\title{
OPTIMAL ITEM CALIBRATION FOR COMPUTERIZED ACHIEVEMENT TESTS
}

\author{
MAHMOOD Ul HASSAN(D) AND FRANK MilleR(D) \\ STOCKHOLM UNIVERSITY
}

\begin{abstract}
Item calibration is a technique to estimate characteristics of questions (called items) for achievement tests. In computerized tests, item calibration is an important tool for maintaining, updating and developing new items for an item bank. To efficiently sample examinees with specific ability levels for this calibration, we use optimal design theory assuming that the probability to answer correctly follows an item response model. Locally optimal unrestricted designs have usually a few design points for ability. In practice, it is hard to sample examinees from a population with these specific ability levels due to unavailability or limited availability of examinees. To counter this problem, we use the concept of optimal restricted designs and show that this concept naturally fits to item calibration. We prove an equivalence theorem needed to verify optimality of a design. Locally optimal restricted designs provide intervals of ability levels for optimal calibration of an item. When assuming a two-parameter logistic model, several scenarios with D-optimal restricted designs are presented for calibration of a single item and simultaneous calibration of several items. These scenarios show that the naive way to sample examinees around unrestricted design points is not optimal.
\end{abstract}

Key words: achievement tests, computerized tests, item calibration, optimal restricted design, twoparameter logistic model.

\section{Introduction}

Achievement tests are an important part, e.g., of higher education to quantify the proficiency of examinees. An alternative of growing importance to traditional paper-and-pencil tests is computerized adaptive tests (CAT). Examinees perform the achievement tests at the computer and everyone receives a sequence of questions, called items. The advantage of CAT is that the items received can depend on the answer to previous items, e.g., examinees with many correct answers can be given more difficult questions subsequently which can then characterize their ability in more detail. By this, questions which are too hard or too easy for an examinee are avoided and "a high-quality estimate of the examinee's proficiency can be made using as few as half as many items than in a fixed-form test" (Buyske, 2005).

A prerequisite before administrating a CAT is the existence of a collection of items, an item bank. Based on the item bank, the CAT-algorithm can choose appropriate items for the examinees. This means that the characteristics of items, e.g., the difficulty, need to be determined before they are included into a CAT. This determination of item characteristics is called calibration of items. A common situation is that achievement tests are done periodically, e.g., year by year. Then the task is to update an item bank continuously with new items. Zheng (2014) pointed out the importance of this item replenishment and stressed the need for efficient and accurate calibration of the new items.

In principle, one could perform separate calibration studies where some voluntary test takers answer to new items. However, this is usually a quite costly option and it can be more feasible to

Electronic supplementary material The online version of this article (https://doi.org/10.1007/s11336-01909673-6) contains supplementary material, which is available to authorized users.

Correspondence should be made to Mahmood Ul Hassan, Stockholm University, Stockholm, Sweden. Email: scenic555@gmail.com 
add instead a small calibration part to an ordinary achievement test. The items from the calibration part are then available in achievement tests in future examination periods. This principle is, e.g., applied in the Swedish Scholastic Assessment Test (Universitets- och högskolerådet, 2019) which is administered as paper-and-pencil test. Adding similarly new items to be calibrated to a CAT has been called online calibration (Stocking, 1988) and Zheng (2014) reviews methods for it. Irrespectively if added to a paper-and-pencil, to a CAT, or to a non-adaptive computerized test, the calibration part has to be quite small such that the burden of this add-on part on the examinees is negligible.

We assume that an ordinary computerized test is performed (CAT or non-adaptive) and that the abilities of the examinees are well determined by their answers to a larger part of the operational items. We focus here in this work on the calibration part for new items which are seeded into the later part of operational items in a computerized test. A set of new items should be tested in the calibration and we consider here the situation that we can allocate to each examinee a small, fixed number of these new items. Our aim is to allocate these items to examinees in a good way such that we obtain high-quality estimates for the item characteristics.

For designing the calibration part, we will apply optimal design theory, see e.g., Atkinson, Donev and Tobias (2007). The use of optimal design theory for item calibration has been discussed previously and designs have been elaborated, see e.g., Berger (1992), Buyske (2005), Lu (2014), Zheng (2014), van der Linden and Ren (2015), Ren, van der Linden and Diao (2017).

In contrast to problems in traditional optimal design setup, we have in this context not the possibility to select examinees with desired proficiency freely within a design space. This would theoretically require the access to a large number of examinees with specific abilities, a problem discussed, e.g., by Zheng (2014), van der Linden and Ren (2015) and Ren et al. (2017). The problem is avoided if sequential optimization is done. Then, for a given examinee, the best calibration item is chosen. Some achievement tests, however, test examinees in parallel and a sequential optimal design cannot be applied. In the Swedish Scholastic Assessment Test, for example, more than 60,000 examinees participate on each of two test dates per year. We consider here such a parallel testing situation, where we have at one test date a given population of examinees for the item calibration: the examinees participating in the computerized test. Based on an assumed proficiency distribution of these examinees, we will apply in this work restricted optimization to this distribution. Restricted optimization (also called constrained or bounded optimization) has been discussed in other contexts than achievement tests by Wynn (1982), Sahm and Schwabe (2000). This type of restricted optimal designs has to our knowledge not been applied for item calibration despite that it is the natural adaption of traditional optimal design to finite populations (Wynn, 1982). We are able with this method to gain general insights how item calibration can be optimized.

In the following Sect. 2, we describe the assumed model and the optimal design theory used. We will then present a new equivalence theorem which provides us with a condition to check whether a certain restricted design is optimal or not. This theorem is very general, e.g., applies to general item response models. In Sect. 2, we also describe the algorithm developed for computation of optimal designs. In Sects. 3 and 4, we compute optimal designs in several scenarios. In these scenarios, we present situations with up to three items to calibrate. In real applications, the number of items usually is much larger. We discuss in Sect. 5 an easy way to apply our results for realistic situations. We summarize our insights and conclude with a discussion (Sect. 6) where we point out directions of future research. The proof of our equivalence theorem is elaborated in an "Appendix". 


\section{Model for Optimal Item Calibration}

\subsection{Model for Item Calibration}

Item response theory (IRT) modeling has been shown to be a flexible tool for item calibration. The idea of IRT is the assumption that each examinee has an ability $\theta \in \Theta=\mathbb{R}$ and that the probability for an examinee with ability $\theta$ to correctly response to item $i(i=1, \ldots, n)$ follows a non-decreasing function

$$
p_{i}(\theta)=P\left(\mathrm{Y}=1 \mid \theta, \beta_{i}\right),
$$

where $Y=1$ indicates a correct and $Y=0$ an incorrect response to an item. The function $p_{i}$ depends on a parameter vector $\beta_{i}$. An assumption which often is made is $\theta \sim N(0,1)$ for the population of examinees. The basic goal of item calibration is to establish a bank of items with known item parameters $\beta_{i}$. The efficiency of an item calibration depends on prior knowledge of item parameters $\left(\beta_{i}\right)$ and ability levels of examinees $(\theta)$ sampled from the population who are to be allocated to the item.

Example 1. An important IRT model used in practice for optimal item calibration is the twoparameter logistic model. The probability for an examinee with ability $\theta$ to correctly response to item $i(i=1, \ldots, n)$ with item parameters $\beta_{i}=\left(a_{i}, b_{i}\right)$ is defined as

$$
p_{i}(\theta)=P\left(\mathrm{Y}=1 \mid \theta, a_{i}, b_{i}\right)=\frac{1}{1+e^{-a_{i}\left(\theta-b_{i}\right)}},
$$

where $a_{i} \in(0, \infty)$ is called discrimination parameter, and $b_{i} \in \mathbb{R}$ is called difficulty parameter of an item. In practice, typical ranges of item parameters might be $b_{i} \in[-3,3], a_{i} \in[0.3,3]$, see Buyske (2005).

\subsection{Optimal Unrestricted Design}

A design for item calibration is a rule how to sample desired ability levels of examinees for estimation of unknown item parameters. We have here $n$ different items to calibrate and assume that each examinee can calibrate at most one of those (see Sect. 5 for the case when each examinee calibrates $k>1$ items). First, we are interested in unrestricted designs, meaning that we have no restrictions in availability of examinees with specific ability levels; the space of examinees' abilities is called $\Theta=\mathbb{R}$. Using continuous designs [see Chapter 9 in Atkinson et al. (2007)], we represent designs by probability measures $\xi$ over the design space $\chi=\Theta \times\{1, \ldots, n\}$. A $(\theta, i) \in \chi$ means here that examinees with ability $\theta$ are sampled for item $i$. The restriction $\xi_{i}$ of $\xi$ to $\Theta \times\{i\}$ describes how abilities of examinees should be chosen for item $i$.

We assume to sample examinees with $m_{i}$ distinct ability levels $\theta_{i 1}, \theta_{i 2}, \ldots, \theta_{i m_{i}}$ in $\Theta$ with sample proportions (weights) $w_{i 1}, \ldots, w_{i m_{i}} \geq 0$ for all items $i$, such that $\sum_{i=1}^{n} \sum_{j=1}^{m_{i}} w_{i j}=1$. Here, $w_{i j}$ is the sample proportion of examinees assigned to each distinct ability level $\theta_{i j}$ for item $i$ and $\sum_{j=1}^{m_{i}} w_{i j}$ is the proportion of examinees assigned to item $i$.

In order to search for a good item calibration design $\xi$, we follow classical optimal design theory and focus on the design's Fisher information matrix for the item parameters. This matrix indicates the precision of the model parameters estimators.

The model (1) is a generalized linear model (GLM). Its logit link $\eta_{i}(\theta)=\log \left(\frac{p_{i}(\theta)}{1-p_{i}(\theta)}\right)$ is assumed to be differentiable in $\beta_{i}$. Further, we assume that $\frac{\partial \eta_{i}(\theta)}{\partial \beta_{i}}$ are continuous in $\theta$. The 
standardized information matrix of item parameters $\beta=\left(\beta_{1}, \ldots, \beta_{n}\right)$ (Fisher's information matrix divided by number of observations) is a block-diagonal matrix

$$
M(\xi)=\operatorname{diag}\left(M_{1}\left(\xi_{1}\right), \ldots, M_{n}\left(\xi_{n}\right)\right) \text { with } M_{i}\left(\xi_{i}\right)=\sum_{j=1}^{m_{i}} w_{i j} v_{i}\left(\theta_{i j}\right)\left(\frac{\partial \eta\left(\theta_{i j}\right)}{\partial \beta_{i}}\right)\left(\frac{\partial \eta\left(\theta_{i j}\right)}{\partial \beta_{i}}\right)^{T}
$$

where $v_{i}(\theta)=p_{i}(\theta)\left(1-p_{i}(\theta)\right)$ is the weight function for this GLM [see Chapter 22 in Atkinson et al. (2007)].

In optimal design theory, we optimize some appropriate convex function $\Psi$ of $M(\xi)$. A design $\xi^{*}$ is called $\Psi$-optimal if $\xi^{*}=\arg \min _{\xi} \Psi\{M(\xi)\}$. The information matrix $M(\xi)$ depends on the unknown model parameters $\beta_{i}, i=1, \ldots, n$. If a researcher has a best guess or initial values about the model parameters, the optimal design can be constructed based on these initial values. Such an optimal design is referred to as a locally $\Psi$-optimal design (Atkinson et al., 2007).

We will consider directional derivatives which tell how the information of a design $\xi$ changes in a direction of another design $\lambda$ :

$$
F_{\Psi}(\xi, \lambda)=\lim _{\alpha \downarrow 0} \frac{1}{\alpha}[\Psi\{M((1-\alpha) \xi+\alpha \lambda)\}-\Psi\{M(\xi)\}]
$$

Especially, when $\lambda$ is the measure $\delta_{(\theta, i)}$ with unit weight at design point $\theta$ for item $i$, we can quantify how much the criterion changes when a small amount of observations in $\theta$ are added for item $i$. We write then $F_{\Psi}(\xi, \theta, i)=F_{\Psi}\left(\xi, \delta_{(\theta, i)}\right)$. An optimality criterion $\Psi$ is called differentiable if all directional derivatives can be expressed as integral over directional derivatives with respect to $\delta_{(\theta, i)}: F_{\Psi}(\xi, \lambda)=\int_{\chi} F_{\Psi}(\xi, \theta, i) \lambda(d(\theta, i))$, see e.g., Whittle (1973). We assume in this paper that criterion $\Psi$ is differentiable.

The General Equivalence Theorem (Kiefer \& Wolfowitz, 1960) is an important result which gives us a way to check whether a design is $\Psi$-optimal among all designs. It states the equivalence of the following three conditions for the design $\xi^{*}$ :

- The design $\xi^{*}$ minimizes $\Psi\{M(\xi)\}$.

- The minimum over $(\theta, i) \in \chi$ of $F_{\Psi}\left(\xi^{*}, \theta, i\right) \geq 0$.

- The minimum over $(\theta, i) \in \chi$ of $F_{\Psi}\left(\xi^{*}, \theta, i\right)=0$ and it is achieved at the support-points $(\theta, i)$ of the design $\xi^{*}$.

Several optimality criteria $\Psi$ (e.g., E-, A-, G- and D-optimality) have been proposed in the literature (Pukelsheim, 2006). The D-optimality criterion is one of the most popular and intensively studied criteria in optimal design methodology (Berger, 1992; Silvey, 1980; Berger, King \& Wong, 2000). It is also most frequently used in online items calibration literature (Chang \& Lu, 2010; Jones \& Jin, 1994; Zhu, 2006). Buyske (1998) has justified the use of a specific L-optimality to reduce parameter drift in online calibration. From the above-mentioned criteria, A-, D-, and L-optimalities are differentiable.

Example 2. We consider again the important two-parameter logistic model (2) and present link function, derivative, information matrix, and directional derivative for D-optimality, since we will illustrate our method with this model in Sects. 3 and 4. The link function is: $\eta_{i}(\theta)=a_{i}\left(\theta-b_{i}\right)$ having the derivative

$$
\frac{\partial \eta_{i}(\theta)}{\partial \beta_{i}}=\left[\begin{array}{c}
\frac{\partial \eta_{i}(\theta)}{\partial a_{i}} \\
\frac{\partial \eta_{i}(\theta)}{\partial b_{i}}
\end{array}\right]=\left[\begin{array}{c}
\left(\theta-b_{i}\right) \\
-a_{i}
\end{array}\right] \text { where } \beta_{i}=\left(a_{i}, b_{i}\right)
$$


The information matrix is:

$$
M_{i}\left(\xi_{i}\right)=\sum_{j=1}^{m_{i}} w_{i j} v_{i}\left(\theta_{i j}\right)\left[\begin{array}{cc}
\left(\theta_{i j}-b_{i}\right)^{2} & -a_{i}\left(\theta_{i j}-b_{i}\right) \\
-a_{i}\left(\theta_{i j}-b_{i}\right) & a_{i}^{2}
\end{array}\right] .
$$

We use the D-optimality criterion in Sects. 3 and 4 which maximizes the determinant of the standardized information matrix $M(\xi)$ so that the generalized variance of the parameter estimates is minimized (Atkinson et al., 2007). Equivalently to maximizing the determinant $|M(\xi)|$ we can minimize $\Psi\{M(\xi)\}=-\log |M(\xi)|=-\sum_{i=1}^{n} \log \left|M_{i}\left(\xi_{i}\right)\right|$ and the directional derivative for this criterion is then given by

$$
F_{D}(\xi, \theta, i)=2-p_{i}(\theta)\left(1-p_{i}(\theta)\right)\left[\left(\theta-b_{i}\right)-a_{i}\right] M_{i}\left(\xi_{i}\right)^{-1}\left[\left(\theta-b_{i}\right)-a_{i}\right]^{T} .
$$

The directional derivative is an important part of the General Equivalence Theorem. Later in Sects. 3 and 4 we use directional derivatives for verifying the optimality of our restricted design with the Equivalence Theorem for Item Calibration which will be presented in Sect. 2.3.

\subsection{Optimal Restricted Design}

Our aim is to select the best subsamples of examinees for each of $n$ new items in order to optimize item calibration. Since we cannot sample a large number of examinees with specific abilities, we cannot apply directly the optimal design based on the method described in Sect. 2.2. However, we can use the main optimal design ideas described before but restrict the set of available designs using an approach initially described by Wynn (1982).

Let $g$ be a continuous density on $\Theta$ which describes the examinees participating in the computerized test. A calibration design is described by sub-densities $h_{0}, h_{1}, \ldots, h_{n} \geq 0$ on $\Theta$ where $h_{1}, \ldots, h_{n}$ describe the sub-population of examinees to be assigned to Item $1, \ldots, n$, respectively, and $h_{0}$ describes the non-sampling distribution. These sub-densities $h_{i}$ should describe together the whole available population $g$ :

$$
\sum_{i=0}^{n} h_{i}(\theta)=g(\theta) \text { for all } \theta \in \Theta .
$$

Further, we allow to use the proportion $s \in(0,1]$ of examinees for calibration (where $s$ can be 1 if there are several items to calibrate, $n \geq 2$ ). This restriction means for the non-sampled population $h_{0}$ :

$$
\int_{\Theta} h_{0}(\theta) \mathrm{d} \theta=1-s
$$

We collate the densities $h_{0}, h_{1}, \ldots, h_{n}$ into a single density $h$ on $\tilde{\chi}=\Theta \times\{0,1, \ldots, n\}$ defined by $h(\theta, i)=h_{i}(\theta)$. The density $h$ defines a probability measure $\xi$ on $\tilde{\chi}$ by $\xi(A)=\int_{A} h(x) \mathrm{d} x$ for sets $A \in \tilde{\chi}$, but we use here in our notation $h$ to represent the design.

Let $\Xi_{s}^{g}$ be the set of all such designs $h$ where the $h_{i}$ fulfill restrictions (3) and (4). The standardized information matrices for item $i$ of a design $h_{i}(i=1, \ldots, n)$ are given by

$$
M_{i}\left(h_{i}\right)=\int_{\Theta} p_{i}(\theta)\left(1-p_{i}(\theta)\right)\left(\frac{\partial \eta(\theta)}{\partial \beta_{i}}\right)\left(\frac{\partial \eta(\theta)}{\partial \beta_{i}}\right)^{T} \frac{h_{i}(\theta)}{s} \mathrm{~d} \theta .
$$


We now search a locally $\Psi$-optimal design in the set of restricted designs, $\Xi_{s}^{g}$. Like in the unrestricted case (see Sect. 2.2), we can characterize an optimal design using an equivalence theorem. We derive a new equivalence theorem for the case of calibration of multiple items. This equivalence theorem uses again a directional derivative $F_{\Psi}(h, \theta, i)$ for design $h$ in direction of the one-point measure in $(\theta, i)$.

For a given design $h^{*}$, we define the minimum $\tilde{L}$ over, the directional derivatives: $\tilde{L}\left(h^{*}, \theta\right)=$ $\min _{i=1, \ldots, n} F_{\Psi}\left(h^{*}, \theta, i\right)$. Further, we define for a given sampling proportion $s$

$$
c^{*}=\arg \max _{c}\left\{\int_{\Theta} \mathbf{1}_{\tilde{L}\left(h^{*}, \theta\right) \leq c} g(\theta) \mathrm{d} \theta \leq s\right\},
$$

where $\mathbf{1}_{A}$ is the indicator function being 1 on a set $A$ and 0 otherwise. Let $L$ be the at $c^{*}$ truncated function $\tilde{L}, L\left(h^{*}, \theta\right)=\min \left\{\tilde{L}\left(h^{*}, \theta\right), c^{*}\right\}$. When formally defining $F_{\Psi}\left(h^{*}, \theta, 0\right)=c^{*}$, we can write

$$
L\left(h^{*}, \theta\right)=\min _{i=0,1, \ldots, n} F_{\Psi}\left(h^{*}, \theta, i\right)
$$

Theorem 1. (Equivalence Theorem for Item Calibration) Let $h^{*} \in \Xi_{s}^{g}$ be a design and $c^{*}$ and $L$ be defined according to (5) and (6) and let $\Psi$ be differentiable. Then: $h^{*}$ is $\Psi$-optimal in $\Xi_{S}^{g}$ if and only if

$$
F_{\Psi}\left(h^{*}, \theta, i\right)=L\left(h^{*}, \theta\right) \text { for } h^{*} \text {-almost all }(\theta, i) \in \tilde{\chi}
$$

We provide a formal proof in "Appendix A".

The use of this equivalence theorem in applications is: For checking if a given candidate design $h^{*}$ is optimal, compute and plot the $n$ directional derivatives $F_{\Psi}\left(h^{*}, \theta, i\right), i=1, \ldots, n$ over $\Theta$. The design is optimal if the sampling is only for items when their directional derivative is smallest and (in case $s<1$ ) if it is below some constant $c^{*}$ which separates the regions of sampling (dir. dev. $\leq c^{*}$ ) from the regions of non-sampling (dir. dev. $\geq c^{*}$ ). We will use this theorem for the examples in Sects. 3 and 4.

A consequence of the theorem is that the optimal design usually samples the full available population on ability intervals for a single item. Only if two (or more) directional derivatives coincide on an interval, it can be optimal to sample these two (ore more) items for the same ability interval.

Example 3. We focus now specifically on D-optimality, $\Psi\{M(\xi)\}=-\log |M(\xi)|$ and the twoparameter logistic model (2). Sahm and Schwabe (2000) argue for the logistic regression model, that $F_{D}(h, \cdot, i), i \neq 0$, has at most three local extrema for all designs $h$ : up to two local minima and one local maximum. Further, $F_{D}(h, \theta, i)<2$ for all $\theta \in \Theta, \lim _{\theta \rightarrow \pm \infty} F_{D}(h, \theta, i)=2$, and $F_{D}(h, \cdot, i)$ is a continuous function and not constant on any interval. From these results mentioned by Sahm and Schwabe (2000), we can conclude in the case of a single item to calibrate, $n=1$, that one can search the optimal design specifically in the designs which sample everyone in (at most) two intervals,

$$
\left[\theta_{1 L}, \theta_{1 U}\right] \cup\left[\theta_{2 L}, \theta_{2 U}\right] \text { with }-\infty<\theta_{1 L} \leq \theta_{1 U} \leq \theta_{2 L} \leq \theta_{2 U}<\infty
$$

and no one outside these intervals. This design has sub-probability density $h_{1}(\theta)=g(\theta)$. $\mathbf{1}_{\left[\theta_{1 L}, \theta_{1 U}\right] \cup\left[\theta_{2 L}, \theta_{2 U}\right]}(\theta)$. The shape of the directional derivatives discussed above tells specifically 
for the case $s<1$ that it is (from a theoretical point of view) never D-optimal to sample the examinees with the lowest and with the highest ability. However, we will see in examples that the optimal design chooses sometimes examinees with very low or very high abilities. If $s=1$, one can show that the D-optimal design allocates examinees with the lowest and the highest ability to the item with the lowest discrimination $a_{i}$.

\subsection{Optimization Algorithm}

The optimization algorithm for optimal restricted designs for the one and two item case is presented below. An idea how to extent to larger numbers of items $n$ will be visible from the case $n=2$-however, complexity will increase.

2.4.1. Optimization Algorithm for $n=1$ Item For the two-parameter logistic model, a twointerval design is optimal. The standard routine for the construction of locally D-optimal twointerval designs (8) is summarized as:

- Step 1: Choose a starting design $h^{0}=g \cdot \mathbf{1}_{\left[\theta_{1 L}^{0}, \theta_{1 U}^{0}\right] \cup\left[\theta_{2 L}^{0}, \theta_{2 U}^{0}\right]}$ which has density $g$ on two intervals $\left[\theta_{1 L}^{0}, \theta_{1 U}^{0}\right]$ and $\left[\theta_{2 L}^{0}, \theta_{2 U}^{0}\right]$ and density 0 otherwise. One may choose the intervals in the starting design around the optimal unrestricted design points which are shown in Sect. 3.

- Step 2: Solve the constrained optimization problem: maximize $|M(\xi)|$ or minimize $-\log |M(\xi)|$ for parameters $\theta_{1 L}, \theta_{1 U}, \theta_{2 L}, \theta_{2 U}$

subject to equality constraint $\int_{\theta_{1 L}}^{\theta_{1}} g(\theta) \mathrm{d} \theta+\int_{\theta_{2 L}}^{\theta_{2} U} g(\theta) \mathrm{d} \theta=s$ [based on (3) and (4)] and

subject to inequality constraint $\theta_{1 L} \leq \theta_{1 U} \leq \theta_{2 L} \leq \theta_{2 U}$.

- Step 3: Finally for assurance, check whether this two-interval design is really D-optimal by computing the directional derivative of the D-criterion and by checking whether the condition in the Equivalence Theorem for Item Calibration is fulfilled.

Note that the equality constraint is not required in Step 1; Step 2 will then ensure it.

2.4.2. Optimization Algorithm for $n=2$ Items $\quad$ If $n=2$ or larger, the number of intervals needed for each item is not necessarily bounded by 2 . We start with allowing $K=2$ intervals for each item in any possible order, determine the restricted D-optimal design (given the additional restriction of up to $K$ intervals) and check with the Equivalence Theorem for Item Calibration if the design is optimal. If it is not optimal, we repeat these steps for $K+1$. In more detail:

- Step 1: Choose a starting design $\xi^{0}$ which has density $g$ on $K$ intervals

for 1st item: $I_{11}=\left[\theta_{11 L}^{0}, \theta_{11 U}^{0}\right], \ldots, I_{1 K}=\left[\theta_{1 K L}^{0}, \theta_{1 K U}^{0}\right]$, for 2nd item: $I_{21}=\left[\theta_{21 L}^{0}, \theta_{21 U}^{0}\right], \ldots, I_{2 K}=\left[\theta_{2 K L}^{0}, \theta_{2 K U}^{0}\right]$ and density 0 otherwise.

- Step 2: Solve the constrained optimization problem: maximize $|M(\xi)|=\left|M_{1}\left(\xi_{1}\right)\right|$. $\left|M_{2}\left(\xi_{2}\right)\right|$ or minimize

$$
-\log \left|M_{1}\left(\xi_{1}\right)\right|-\log \left|M_{2}\left(\xi_{2}\right)\right|
$$

subject to equality constraint $\sum_{r=1}^{n} \sum_{t=1}^{K} \int_{I_{r t}} g(\theta) \mathrm{d} \theta=s$ and 
subject to inequality constraint $I_{11} \ldots I_{1 K} I_{21} \ldots I_{2 K} \Leftrightarrow \theta_{11 L} \leq \theta_{11 U} \leq \cdots \leq$ $\theta_{12 L} \leq \theta_{12 U} \leq \theta_{21 L} \leq \theta_{21 U} \leq \cdots \leq \theta_{22 L} \leq \theta_{22 U}$.

Similarly we check other possible ordering of intervals. For $K=2$, we have six inequalities constraints $I_{11} I_{12} I_{21} I_{22}, I_{11} I_{21} I_{12} I_{22}, I_{11} I_{21} I_{22} I_{12}, I_{21} I_{22} I_{11} I_{12}, I_{21} I_{11} I_{22} I_{12}$, $I_{21} I_{11} I_{12} I_{22}$. We select the inequity constraint which gives minimum value in (9).

- Step 3: Finally for assurance, it is essential to check whether this $K$-interval design is really D-optimal by computing the directional derivative of the $\mathrm{D}$-criterion and by checking whether the condition in the Equivalence Theorem for Item Calibration is fulfilled. If the design is not optimal, set $K=K+1$ and go to Step 1 .

The number of inequalities constraints is generally $\left(\begin{array}{c}2 K \\ K\end{array}\right)$. In the computations we made for the examples in Sect. 4, it was sufficient to use $K=2$ and $K=3$.

Since it is allowed that two or more interval boundaries coincide, the designs which need less than $K$ intervals are special cases of a $K$-interval design. Hence, when increasing $K$, (9) cannot increase; it is decreasing until the right $K$ is found and would then be constant for larger $K$. If some interval boundaries coincide in the determined optimal design, we can finally reduce the number of intervals of it.

The constrained optimization problem in our algorithm was solved by using the R-package nloptr (Borchers, 2013) with Sequential (least-squares) Quadratic Programming (SQP) algorithm. We use the algorithm for the examples presented in Sects. 3 and 4. The number of iterations of the SQP algorithm can vary considerably for each case, but a final solution is obtained in all cases very quickly within one minute.

\subsection{Relative Efficiency of Designs}

The relative D-efficiency $\left(\mathrm{RE}_{\mathrm{D}}\right)$ of a design $h^{(1)}$ compared to another design $h^{(2)}$ is

$$
\mathrm{RE}_{\mathrm{D}}=\mathrm{RE}_{\mathrm{D}}\left(h^{(1)}, h^{(2)}\right)=\left[\frac{\left|M\left(h^{(1)}\right)\right|}{\left|M\left(h^{(2)}\right)\right|}\right]^{\frac{1}{2 n}}=\left[\frac{\prod_{i=1}^{n}\left|M_{i}\left(h_{i}^{(1)}\right)\right|}{\prod_{i=1}^{n}\left|M_{i}\left(h_{i}^{(2)}\right)\right|}\right]^{\frac{1}{2 n}}
$$

where $2 n$ is the number of parameters, see Berger and Wong (2009). An $R_{D_{D}}$-value less than 1 indicates that design $h^{(2)}$ is better than design $h^{(1)}$ in terms of D-optimality. In terms of sample size, this means that design $h^{(1)}$ approximately needs $\left(\mathrm{RE}_{\mathrm{D}}^{-1}-1\right) * 100 \%$ more examinees to be as efficient as $h^{(2)}$.

We could assign the items randomly irrespectively of the ability such that each examinee has probability $s / n$ to calibrate a specific item. This so-called random design has densities $h_{i}^{r}=s g / n$ for $i=1, \ldots, n$. In the examples, we will be interested, e.g., in the relative efficiency $\operatorname{RE}_{\mathrm{D}}\left(h^{r}, h^{*}\right)$ of the random design compared to the D-optimal restricted design $h^{*}$. Many researchers have compared optimal design efficiency with a random design in item calibration studies, see e.g., Buyske (2005). We consider a random design as a benchmark for comparison.

Another important design for comparison is the symmetric design. It divides first the sample proportion $s$ equally to all, say $m$, unrestricted design points and a proportion $s / m$ should be sampled around a design point $\theta^{*}$. A value $d$ is computed such that the desired proportion $s / m$ of examinees is in the symmetric interval $\left(\theta^{*}-d, \theta^{*}+d\right)$, i.e., $\int_{\theta^{*}-d}^{\theta^{*}+d} g(x) \mathrm{d} x=s / m$. The symmetric design is only well defined as long as the intervals from the design points do not overlap. 


\section{Results for Calibration of One Item}

We consider now three new items to calibrate for our item bank. Assuming a two-parameter logistic model (2), the best guess items parameters $a_{i}$ and $b_{i}$ of these items are:

- Item 1: Discrimination $a_{1}=1$, difficulty $b_{1}=0.5$;

- Item 2: Discrimination $a_{2}=1.5$, difficulty $b_{2}=-1.2$;

- Item 3: Discrimination $a_{3}=1.6$, difficulty $b_{3}=2$.

Usually, the number of items to calibrate is larger than the number which can be allocated to a single examinee. Imagine, e.g., a situation where we have ten times more new items than can be calibrated by an examinee. Focusing first on a specific new item, it should therefore be assigned to approximately $10 \%$ of the examinees. In this section, we illustrate scenarios where we choose a proportion $s<1$ of examinees to calibrate a single item, $n=1$. We ignore in this section that items cannot be treated separately as they might need the same examinees for calibration. In Sects. 4 and 5, we will calibrate then several items simultaneously.

Abdelbasit and Plackett (1983) showed that for the two-parameter logistic model the locally D-optimal design for a single item with best guess values $a$ and $b$ for discrimination and difficulty has two distinct equally weighted design points or ability levels $\theta=b \pm \frac{1.543}{a}$. The corresponding probabilities of correctly answering the question at these points, $\theta_{1}$ and $\theta_{2}$ say, are $p\left(\theta_{1}\right)=0.176$ and $p\left(\theta_{2}\right)=0.824$. This means that the unrestricted locally D-optimal design recommends to choose half of the examinees for calibration with ability $\theta_{1}=b-\frac{1.543}{a}$ and half with ability $\theta_{2}=b+\frac{1.543}{a}$.

We assume in the examples in Sects. 3 and 4 that the examinees in the computerized test have standard normal distributed abilities. We compute locally D-optimal restricted designs with restriction $g(\theta)=\frac{1}{\sqrt{2 \pi}} e^{\left(-\frac{\theta^{2}}{2}\right)}$. However, the method including the Equivalence Theorem in Sect. 2.3 is valid even if another assumption for the abilities is preferred. Since we compute here locally optimal designs, we have made investigations in the supplementary materials where we see robustness as long as the parameters are not severely misspecified.

\subsection{Calibration of Item 1}

In the first scenario, we consider an item with best guess for the difficulty parameter $b=0.5$ and item discrimination parameter $a=1$ (Item 1). We want to select $10 \%$ of the population of examinees to calibrate this item in the item bank. The unrestricted optimal design recommends to sample 5\% examinees with ability level $0.5-\frac{1.543}{1}=-1.043$ and $5 \%$ with $0.5+\frac{1.543}{1}=2.043$. The best guess probability for correct response and the optimal unrestricted design points are shown in the upper panel of Fig. 1a.

It is hard to select a sample of examinees with these specific ability levels as there might be no such examinees available or we have a limited number of examinees with these ability levels. We sample instead the examinees from the available distribution in an optimal way using the techniques described in Sect. 2.3. For the restricted optimal design, we assume that the population of examinees has a standard normal ability distribution and we sample a proportion $s=0.1$ of this population. The calculated optimal restricted design recommends to sample 5\% examinees from the population with ability level between $(-1.215,-0.984)$ and $5 \%$ between $(1.600,2.577)$, see the middle panel of Fig. 1a. The intervals are not equal in length: We have limited available examinees around the high unrestricted ability level 2.043 compared to the low level -1.043 . So we need a longer interval around the unrestricted ability level 2.043 to select 5\% examinees of population. The intervals are also asymmetrical around the unrestricted design points and extend more toward the extreme abilities since less examinees are available there. 


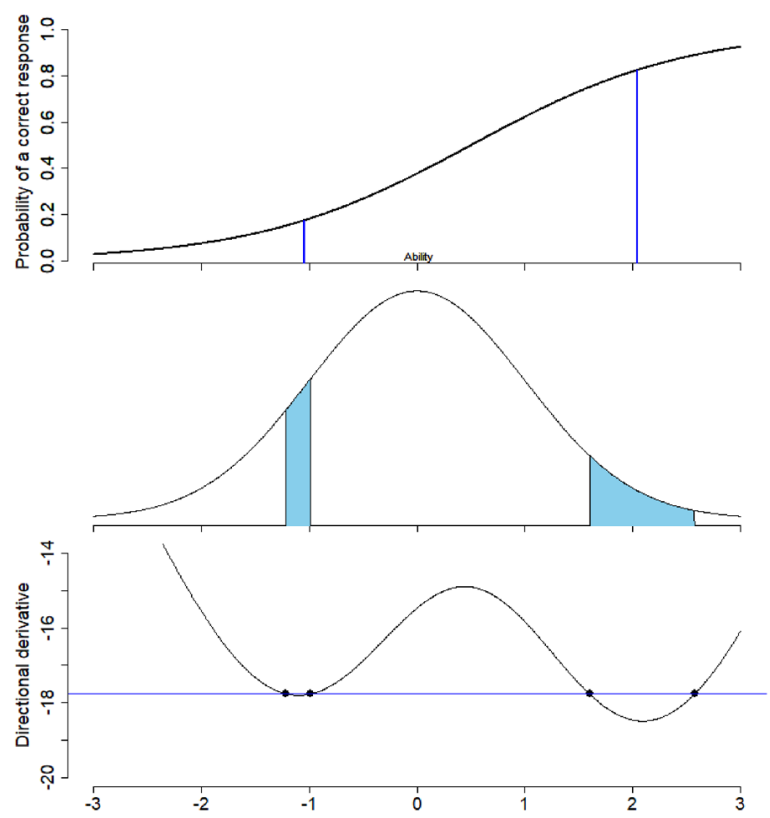

(a) Locally D-optimal unrestricted and restricted $(s=0.1)$ design for calibration of Item 1. Upper panel: black line represents the two-parameter logistic model curve and two blue lines indicate the ability levels of the locally D-optimal unrestricted design; Middle panel: two shaded parts of normal distribution represent the two intervals for ability levels of the locally D-optimal restricted design; Lower panel: Directional derivative (black line) for locally D-optimal restricted design for Item 1, lower and upper interval limits of design (black dots), reference line $c^{*}$ (blue) separating sampling and non-sampling area

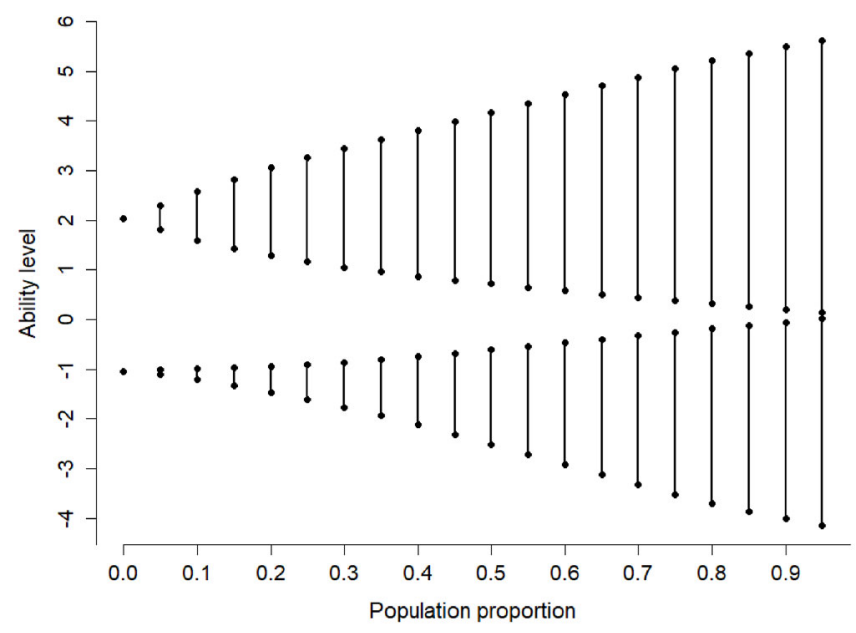

(b) Locally D-optimal restricted designs for $s=0,0.05, \ldots, 0.95$

FIGURE 1.

Locally D-optimal restricted designs for Item 1 (Color figure online). 


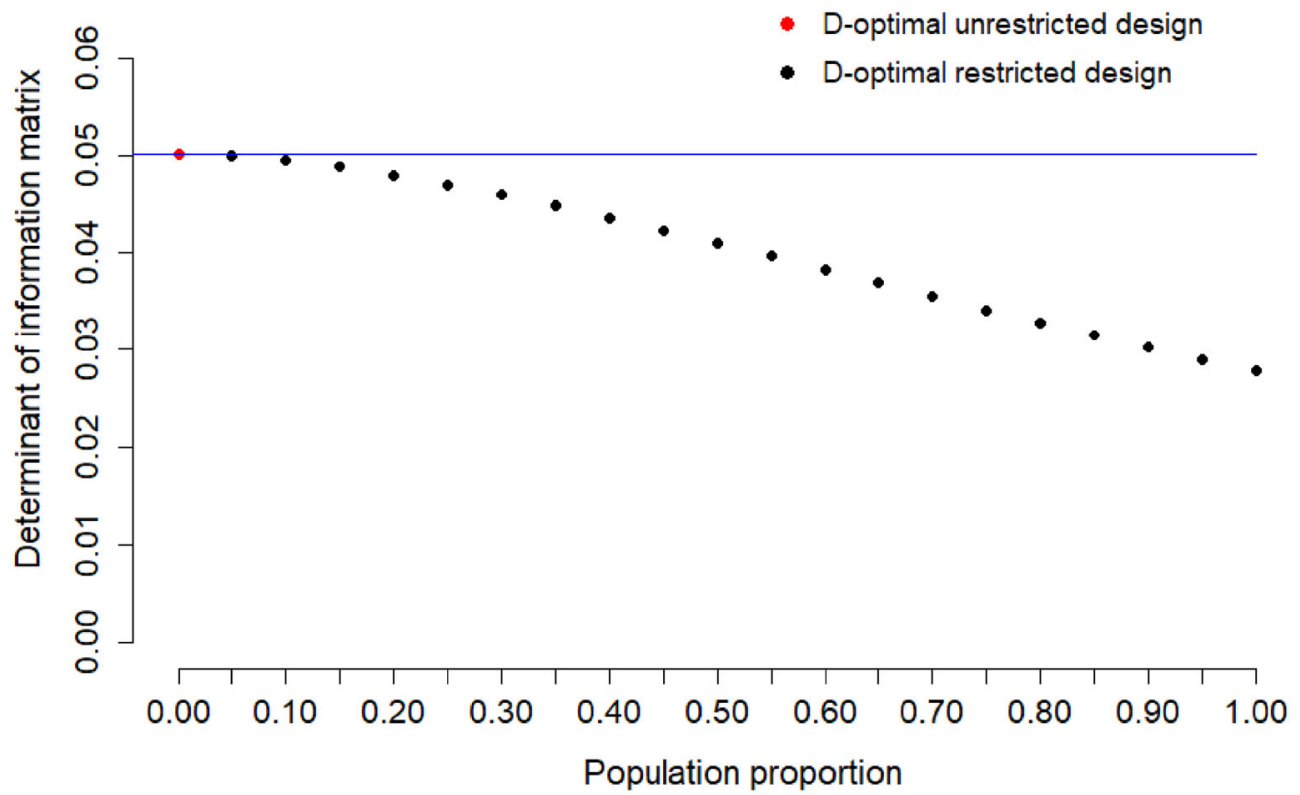

FIGURE 2.

Determinant of information matrix of locally D-optimal restricted design for calibration of Item 1 for sample proportion $s=0,0.05, \ldots, 0.95,1$. The blue line indicates the maximum value of determinant of the information matrix of two-point unrestricted design (Color figure online).

The directional derivative for this two-interval design is shown in the lower panel of Fig. 1a with black line and interval limits are marked on it with red dots. Since these four points of the two-interval design are on one blue reference line and the intervals of the population sample have directional derivative below the reference line, the Equivalence Theorem for Item Calibration described in Sect. 2.3 confirms the optimality of this two-interval design (the blue reference line corresponds to $c^{*}$ in the theorem).

We computed the optimal restricted design for other sample proportions than $s=0.1$. We show the results in Fig. $1 \mathrm{~b}$ for $s=0,0.05,0.1, \ldots, 0.95$, where $s=0$ is the limiting case of unrestricted optimal design. We see there that we still have a two-interval design if we want to sample $95 \%$ of the population. This two-interval design becomes one interval if we sample $96 \%$ of the population. Figure 2 shows the determinant of the information matrix of the locally D-optimal restricted design for Item 1 for sample proportion $s=0,0.05, \ldots, 0.95,1$. The case $s=0$ corresponds to the locally D-optimal unrestricted design, $s=1$ to the random design. The loss of information for Item 1 is moderate if the population proportion is between 0.0 and 0.2 .

\subsection{Calibration of Item 2}

Now we discuss another scenario where we want to calibrate Item 2 with best guess for difficulty parameter $b=-1.2$ and discrimination parameter $a=1.5$. To calibrate this item, we want to sample $25 \%$ of examinees population $(s=0.25)$. The unrestricted design recommends to sample $12.5 \%$ of examinees at each of the ability levels -2.229 and -0.171 . Again we use restricted optimal design because of unavailability or limited available examinees with these specific ability levels. To calibrate this item, we sample $11.48 \%$ from the population of examinees between ability levels $(-3.592,-1.200)$ and $13.52 \%$ between $(-0.061,0.282)$, see Fig. 3a with design and directional derivative plot. In this scenario, the selected sample proportion of the population for the two intervals is not equal. Another remarkable fact is that the upper interval 
not contains the upper value of the optimal unrestricted design. So sampling around this optimal unrestricted design point definitely produces a non-optimal restricted design. Since around $\theta=0$ more examinees are available compared to ability level -2.229 , the upper interval has a shorter length compared to the lower. However in this case, the symmetric design needs only $1.98 \%$ more examinees to have the same efficiency than the restricted D-optimal design, i.e., it is an efficient design; the random design needs $45.45 \%$ more examinees. When investigating other sample proportions $s$, we see that the optimal two-interval design will become a one-interval design if we want to sample a proportion of $90 \%$ or more of the population to calibrate the item. Results for other $s$ are presented in Fig. 3b. For Item 2, we show the determinant of the information matrix of the locally D-optimal restricted design for different sample proportions in Fig. 4. The loss of information for Item 2 is moderate with population proportions between 0.0 and 0.2.

\subsection{Calibration of Item 3}

In the third scenario, we want to sample $35 \%$ of the examinees population $(s=0.35)$ in order to calibrate Item 3 with best guess for difficulty parameter $b=2$ and discrimination parameter $a=1.6$. The unrestricted optimal design recommends to choose $17.5 \%$ sample proportion of the population at each of the ability levels 1.035 and 2.965. The restricted optimal design samples $21.23 \%$ from the population of examinees between ability levels $(0.043,0.611)$ and $13.76 \%$ between $(1.091,5.417)$, see Fig. 5a with design and directional derivative plot. Both intervals have unequal length and different sample proportions of the examinees population. The lower limit of the upper interval is quite close to the lower point of the optimal unrestricted design. This seems reasonable as limited examinees are available around the high ability level 2.965. So to select the examinees this lower limit of the upper interval moves toward the left as more examinees are available to this side. To counter this, the lower interval is quite below from the lower point of the optimal unrestricted design. Similarly as for Item 2, the lower interval does here not contain the lower unrestricted design point. This effect happens for items with difficulty $b$ not in the center of the ability distribution. The value of difficulty where this effect starts depends on the discrimination $a$. In the supplementary materials, we provide figures showing combinations of $a$ and $b$ where an unrestricted design point is not contained in the restricted optimal design.

In terms of sample size, the random design needs $59.66 \%$ more examinees to have the same efficiency as a restricted D-optimal design. If we would try to select examinees symmetrically around the points of the unrestricted design, we have the problem that the intervals around the two design points overlap, i.e., the symmetric design is not usable directly. For sampling proportion $s=0.2$, the symmetric design has no overlapping intervals; in that case, the symmetric design needs $12.95 \%$ more examinees to have the same efficiency than the restricted D-optimal design. Investigating other sample proportions $s$, the optimal two-interval design will become a oneinterval design if we sample a proportion of 55\% or more of the population, see Fig. 5b. Figure 6 indicates that the loss of information is considerable for Item 3 if the population proportion is between 0.0 and 0.2 .

\subsection{Relative Efficiency of the Optimal Design}

Table 1 shows the relative efficiency of the random design versus the D-optimal restricted design for each of the three items. The D-optimal restricted design is generally very efficient compared to the random design gaining up to $34 \%$ sample size for Item 1 , up to $56 \%$ for Item 2 , and $144 \%$ for Item 3 for giving the same precision of estimates. Additionally for the Doptimal restricted and random designs, we provide figures with the determinants of the information matrices for the three items in the supplementary materials. Table 2 shows efficiencies for the symmetric design. For Item 1 which has a difficulty close to the mean ability of the population, the symmetric design is quite efficient needing only up to $1.95 \%$ more examinees compared to the 


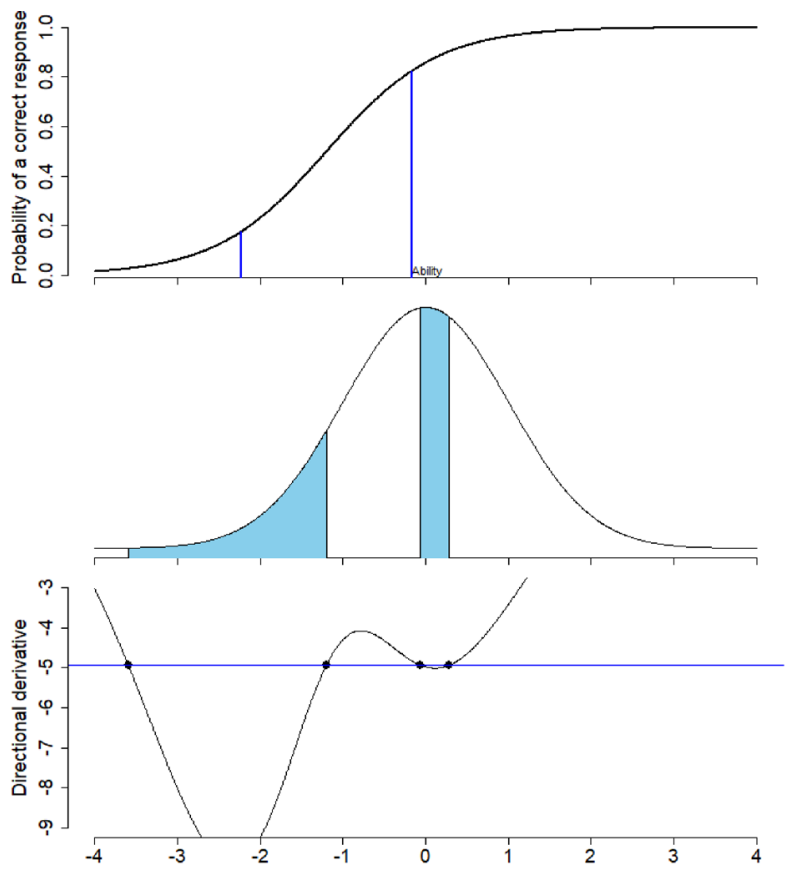

(a) Locally D-optimal unrestricted and restricted $(s=0.25)$ design for calibration of Item 2. See Figure 1a for description of panels.

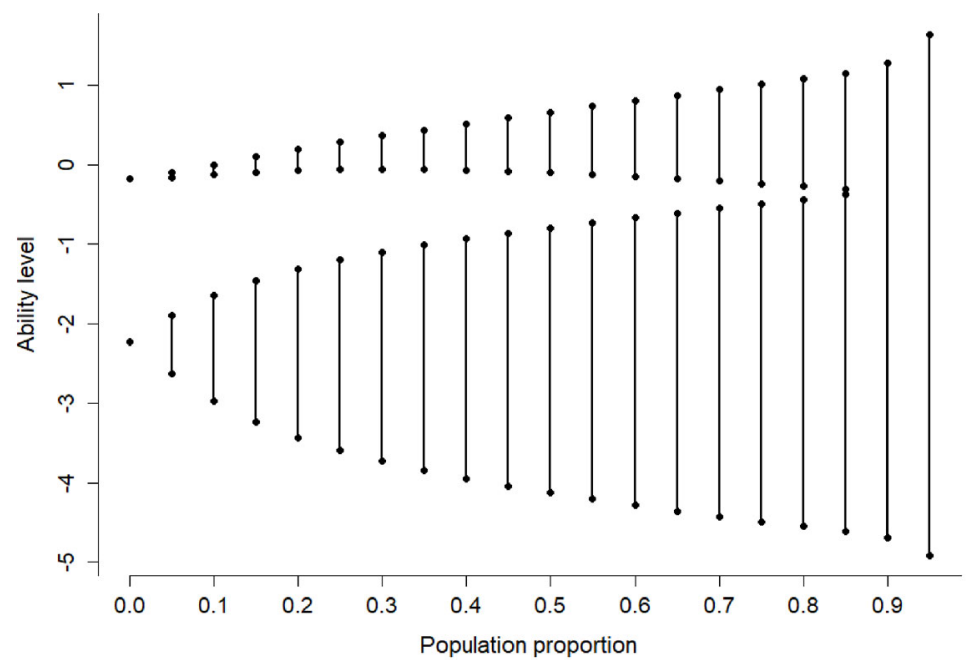

(b) Locally D-optimal restricted designs for $s=0,0.05, \ldots, 0.95$

FIGURE 3.

Locally D-optimal restricted designs for Item 2. 


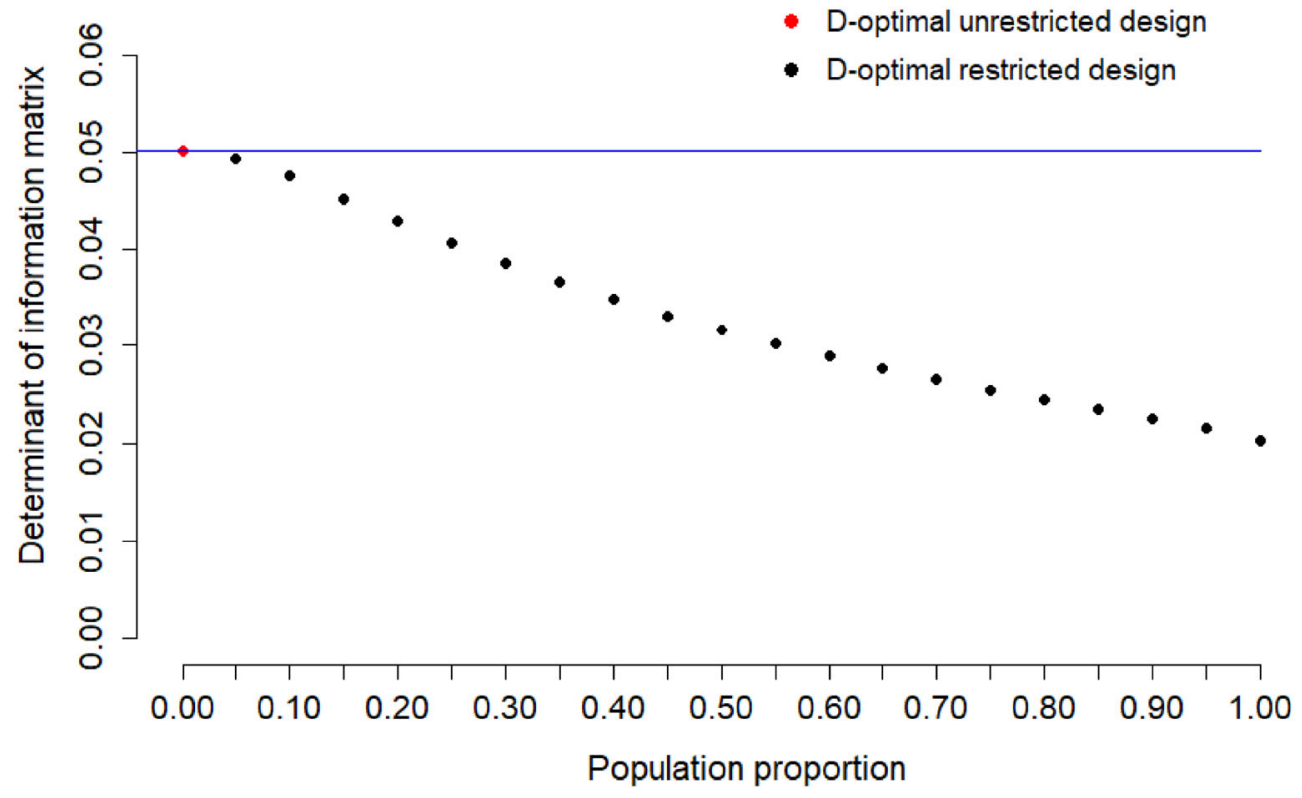

FIGURE 4.

Determinant of information matrix of locally D-optimal restricted design for calibration of Item 2 for sample proportion $s=0,0.05, \ldots, 0.95,1$. The blue line indicates the maximum value of determinant of the information matrix of two-point unrestricted design (Color figure online).

restricted D-optimal design. For Item 2 and 3, the intervals of the symmetric design would overlap for larger $s$; therefore, the design is only possible for $s \leq 0.55$ and $s \leq 0.2$, respectively. For these items having one unrestricted optimal design point where only few examinees are available, there is a higher sample size gain of the optimal compared to the symmetric design for some $s$ (up to $6.80 \%$ for Item 2 and $12.95 \%$ for Item 3).

\section{Results for Calibration of Two or More Items}

We now present scenarios for calibration of two items. We start with briefly mentioning the locally D-optimal unrestricted design. One can show that it is D-optimal to sample exactly half of the examinees for each of the two items. Within each item, the one-item optimal design mentioned in Sect. 3 is the best choice. This means, the locally D-optimal design for calibration of two items is to sample $25 \%$ of the examinees with ability levels $\theta=b_{1} \pm \frac{1.543}{a_{1}}$ for Item 1 and $25 \%$ of the examinees with ability levels $\theta=b_{2} \pm \frac{1.543}{a_{2}}$ for Item 2 .

We compute now locally D-optimal restricted designs assuming that the examinees participating in the computerized test have standard normal distributed abilities. We use Item 1, 2, and 3 from Sect. 3 and compute the optimal design when at least two of these three items should be calibrated simultaneously.

In a first case (Sect. 4.1), the optimal designs for each of the two items are not overlapping. In more challenging cases (see Sects. 4.2 and 4.3), it can be seen that some examinees would be needed for both items - they compete with each other. Then, the optimal design will determine the best allocation to either of the items. The result can be a two-interval solution for both items (Sect. 4.2); in this case, the algorithm in Sect. 2.4.2 found the optimal design using $K=2$. In Sect. $4.3, K=3$ intervals were needed for each item. Finally, we compute optimal designs when 


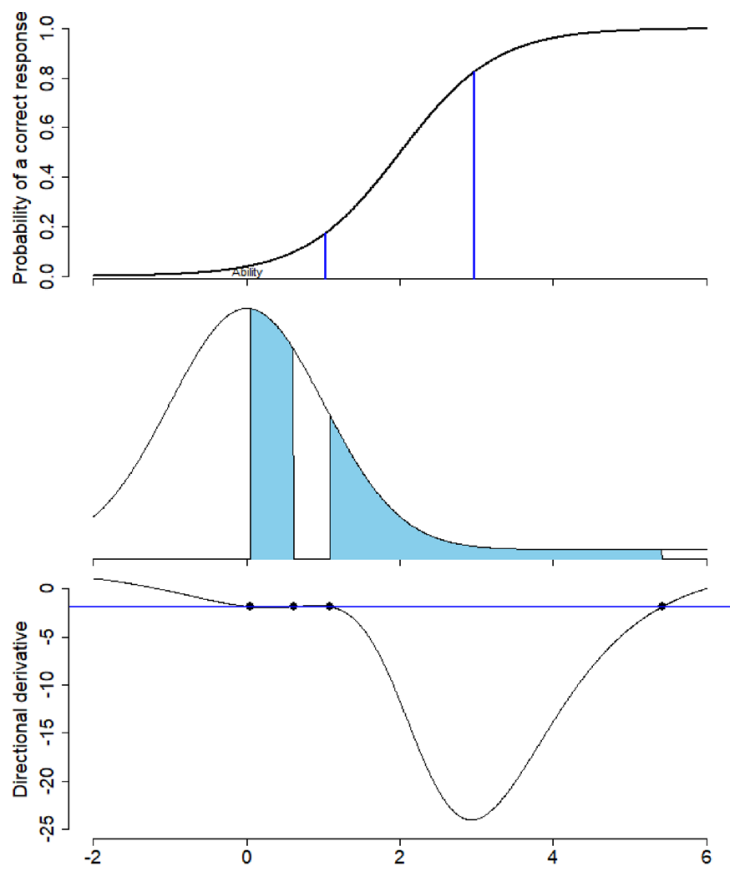

(a) Locally D-optimal unrestricted and restricted $(s=0.35)$ design for calibration of Item 3. See Figure 1a for description of panels.

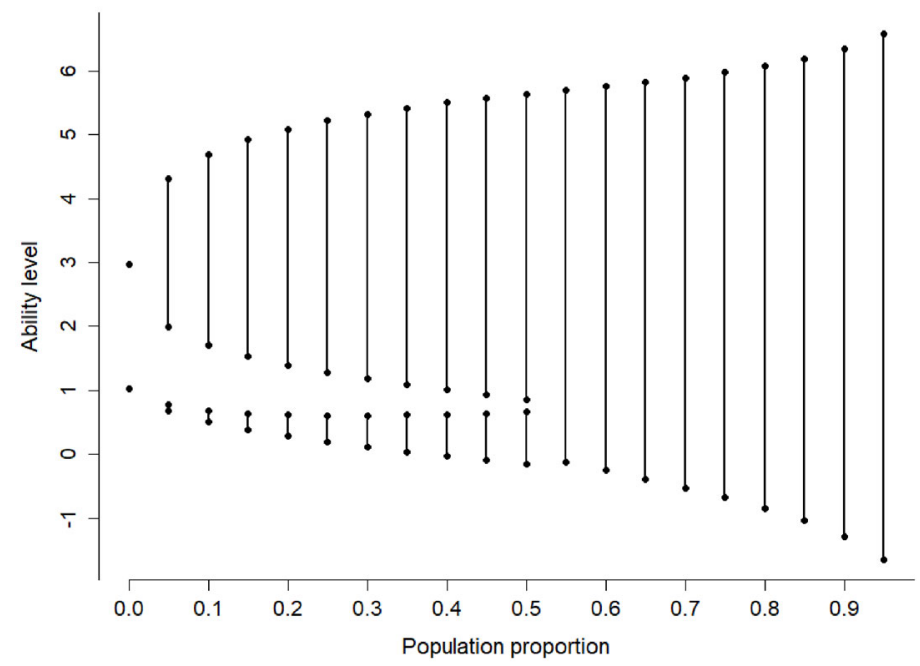

(b) Locally D-optimal restricted designs for $s=0,0.05, \ldots, 0.95$

FIGURE 5.

Locally D-optimal restricted designs for Item 3. 


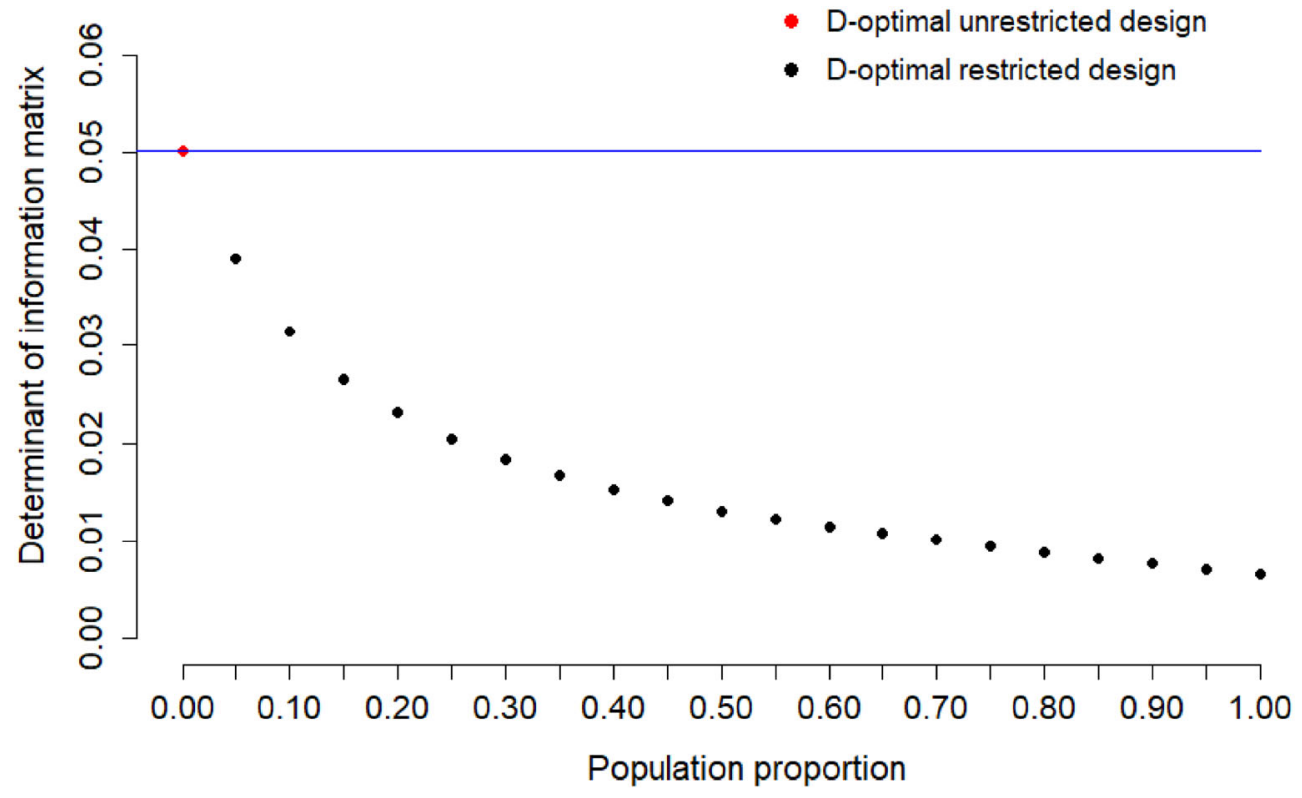

FIGURE 6.

Determinant of information matrix of locally D-optimal restricted design for calibration of Item 3 for sample proportion $s=0,0.05, \ldots, 0.95,1$. The blue line indicates the maximum value of determinant of the information matrix of two-point unrestricted design (Color figure online).

all examinees are sampled ( $s=1$; Sects. 4.4 and 4.5). In Table 3a, the relative efficiencies are calculated for the random design. Considerable sample size gains exist in all cases $(17.30 \%$ to 95.57\%). Table $3 \mathrm{~b}$ shows efficiencies for the symmetric design in cases where intervals do not overlap. We see that in many cases including all cases for calibrating Item 1 and 3, we cannot apply the symmetric design directly due to overlapping.

\subsection{Calibration for Non-competing Items}

In this first situation, we consider Item $1(a=1, b=0.5)$ and Item $2(a=1.5, b=-1.2)$ for calibration. We are interested to sample $10 \%$ population of examinees to calibrate these two items in the item bank.

The unrestricted optimal design suggests to sample $2.5 \%$ examinees at each ability levels $-1.043,2.043$ (for Item 1) and $-2.229,-0.171$ (for Item 2). Since it is in practice hard to sample the examinees at these specific ability levels due to unavailability or limited availability of number of examinees, we use restricted optimal design to sample the examinees between some intervals of ability levels in an optimal way. The unrestricted optimal design recommends us to sample $2.52 \%$ and $2.51 \%$ examinees from the population between ability levels $(-1.114,-1.004)$ and $(1.804,2.308)$, respectively, for Item 1 . For Item 2 , it suggests to choose $2.47 \%$ and $2.50 \%$ of the examinees between ability levels $(-2.617,-1.893)$ and $(-0.167,-0.103)$, see Fig. 7a. The directional derivative plot in the lower panel of Fig. 7a confirms that the design with these intervals limits is optimal: The blue reference line (corresponding to value $c^{*}$ in the Equivalence Theorem for Item Calibration) separates the sampling regions from the non-sampling regions. Further, the sampling to item $i, i=1,2$, corresponds to the region where the respective item has the smallest directional derivative. We show optimal designs for other values of $s \in\{0.1,0.2, \ldots, 1\}$ in Fig. $7 \mathrm{~b}$. 
TABLE 1.

Relative efficiency of random design versus D-optimal restricted design.

\begin{tabular}{|c|c|c|c|c|c|c|}
\hline \multirow[t]{2}{*}{ Proportion (\%) } & \multicolumn{2}{|c|}{ Item 1} & \multicolumn{2}{|c|}{ Item 2} & \multicolumn{2}{|c|}{ Item 3} \\
\hline & $\mathrm{RE}_{\mathrm{D}}$ & $\mathrm{RE}_{\mathrm{SS}}(\%)$ & $\mathrm{RE}_{\mathrm{D}}$ & $\mathrm{RE}_{\mathrm{SS}}(\%)$ & $\mathrm{RE}_{\mathrm{D}}$ & $\mathrm{RE}_{\mathrm{SS}}(\%)$ \\
\hline 0 & 0.7451 & 34.2076 & 0.6360 & 57.2429 & 0.3616 & 176.5380 \\
\hline 5 & 0.7463 & 33.9931 & 0.6407 & 56.0771 & 0.4094 & 144.2796 \\
\hline 10 & 0.7498 & 33.3776 & 0.6531 & 53.1182 & 0.4562 & 119.2079 \\
\hline 15 & 0.7551 & 32.4376 & 0.6695 & 49.3729 & 0.4964 & 101.4377 \\
\hline 20 & 0.7618 & 31.2638 & 0.6875 & 45.4549 & 0.5325 & 87.7791 \\
\hline 25 & 0.7697 & 29.9238 & 0.7062 & 41.6082 & 0.5658 & 76.7462 \\
\hline 30 & 0.7785 & 28.4552 & 0.7251 & 37.9183 & 0.5969 & 67.5348 \\
\hline 35 & 0.7882 & 26.8741 & 0.7440 & 34.4095 & 0.6263 & 59.6613 \\
\hline 40 & 0.7988 & 25.1845 & 0.7629 & 31.0821 & 0.6544 & 52.8130 \\
\hline 45 & 0.8105 & 23.3856 & 0.7817 & 27.9273 & 0.6813 & 46.7767 \\
\hline 50 & 0.8232 & 21.4783 & 0.8004 & 24.9334 & 0.7072 & 41.4018 \\
\hline 55 & 0.8370 & 19.4692 & 0.8191 & 22.0882 & 0.7329 & 36.4384 \\
\hline 60 & 0.8520 & 17.3720 & 0.8377 & 19.3804 & 0.7563 & 32.2257 \\
\hline 65 & 0.8680 & 15.2055 & 0.8562 & 16.8001 & 0.7807 & 28.0906 \\
\hline 70 & 0.8850 & 12.9922 & 0.8746 & 14.3393 & 0.8063 & 24.0296 \\
\hline 75 & 0.9029 & 10.7552 & 0.8929 & 11.9919 & 0.8331 & 20.0334 \\
\hline 80 & 0.9215 & 8.5176 & 0.9111 & 9.7539 & 0.8614 & 16.0870 \\
\hline 85 & 0.9407 & 6.3018 & 0.9292 & 7.6245 & 0.8915 & 12.1685 \\
\hline 90 & 0.9603 & 4.1296 & 0.9472 & 5.5690 & 0.9238 & 8.2444 \\
\hline 95 & 0.9802 & 2.0222 & 0.9692 & 3.1792 & 0.9592 & 4.2536 \\
\hline
\end{tabular}

Relative efficiency $\mathrm{RE}_{\mathrm{D}}$ versus D-optimal restricted design for calibration of one item. $\mathrm{RE}_{\mathrm{SS}}$ indicates sample size gain when using optimal restricted design instead of symmetric design.

\subsection{Calibration for Competing Items}

In this case, we want to select a sample of $50 \%$ examinees from the population in order to calibrate Item $1(a=1, b=0.5)$ and Item $3(a=1.6, b=2)$ in the item bank. The unrestricted optimal design would select $12.5 \%$ examinees each at the ability levels $-1.043,2.043$ for Item 1 and $12.5 \%$ examinees at each ability levels $1.035,2.965$ for Item 3 . Selecting examinees around the unrestricted design points in a naive manner faces the problem that there are only few examinees around the ability levels 2.043 and 2.965 . The restricted design recommends us to choose $15.1 \%$ and $13.6 \%$ of the population of examinees on the ability intervals $(-2.158,-0.967)$ and $(0.836$, $1.511)$ for Item 1 and choose $14.7 \%$ and $6.5 \%$ of the examinees for Item 3 on the intervals $(0.299$, $0.721)$ and $(1.511,5.197)$, see Fig. 8a. The directional derivative plot in the lower panel confirms based on the Equivalence Theorem for Item Calibration that this restricted optimal design is optimal. In each region, the item with the lowest directional derivative is sampled. The two upper intervals follow directly after each other with boundary point 1.511 . This shows that the two items are competing for examinees around this ability $\theta=1.511$. The directional derivative of both items is equal at this point. Examinees with such $\theta$ would be good for both items since both directional derivatives are well below the reference line, but in order to maximize the overall information, this cut-point was determined. Figure $8 \mathrm{~b}$ shows optimal designs for other values of $s \in\{0.1,0.2, \ldots, 1\}$. 
TABLE 2.

Relative efficiency of symmetric design versus D-optimal restricted design.

\begin{tabular}{|c|c|c|c|c|c|}
\hline \multirow[t]{2}{*}{ Proportion (\%) } & \multicolumn{2}{|c|}{ Item 1} & \multirow[t]{2}{*}{ Proportion (\%) } & \multicolumn{2}{|c|}{ Item 2} \\
\hline & $\mathrm{RE}_{\mathrm{D}}$ & $\operatorname{RE}_{S S}(\%)$ & & $\mathrm{RE}_{\mathrm{D}}$ & $\mathrm{RE}_{\mathrm{SS}}(\%)$ \\
\hline 0 & 1.0000 & 0.0000 & 0 & 1.0000 & 0.0000 \\
\hline 5 & 0.9999 & 0.0063 & 5 & 0.9993 & 0.0661 \\
\hline 10 & 0.9994 & 0.0646 & 10 & 0.9954 & 0.4620 \\
\hline 15 & 0.9980 & 0.1990 & 15 & 0.9887 & 1.1451 \\
\hline 20 & 0.9961 & 0.3953 & 20 & 0.9806 & 1.9777 \\
\hline 25 & 0.9937 & 0.6335 & 25 & 0.9722 & 2.8640 \\
\hline 30 & 0.9911 & 0.8937 & 30 & 0.9639 & 3.7426 \\
\hline 35 & 0.9886 & 1.1562 & 35 & 0.9563 & 4.5690 \\
\hline 40 & 0.9862 & 1.4028 & 40 & 0.9496 & 5.3106 \\
\hline 45 & 0.9841 & 1.6155 & 45 & 0.9439 & 5.9429 \\
\hline 50 & 0.9825 & 1.7809 & 50 & 0.9394 & 6.4461 \\
\hline 55 & 0.9814 & 1.8914 & 55 & 0.9363 & 6.8045 \\
\hline 60 & 0.9809 & 1.9450 & & & \\
\hline 65 & 0.9809 & 1.9463 & \multirow[t]{2}{*}{ Proportion (\%) } & \multicolumn{2}{|c|}{ Item 3} \\
\hline 70 & 0.9813 & 1.9018 & & $\mathrm{RE}_{\mathrm{D}}$ & $\mathrm{RE}_{\mathrm{SS}}(\%)$ \\
\hline 75 & 0.9821 & 1.8195 & 0 & 1.0000 & 0.0000 \\
\hline 80 & 0.9832 & 1.7072 & 5 & 0.9633 & 3.8065 \\
\hline 85 & 0.9845 & 1.5726 & 10 & 0.9252 & 8.0835 \\
\hline 90 & 0.9860 & 1.4229 & 15 & 0.8998 & 11.1370 \\
\hline 95 & 0.9875 & 1.2639 & 20 & 0.8853 & 12.9498 \\
\hline
\end{tabular}

Relative efficiency $\mathrm{RE}_{\mathrm{D}}$ versus $\mathrm{D}$-optimal restricted design for calibration of one item. $\mathrm{RE}_{\mathrm{SS}}$ indicates sample size gain when using optimal restricted design instead of symmetric design.

\subsection{Calibration for Items with Several Intervals}

Now in this situation we want to choose a sample of $80 \%$ examinees from the population to calibrate Item $2(a=1.5, b=-1.2)$ and Item $3(a=1.6, b=2)$ in the item bank. The unrestricted design recommends us to choose $20 \%$ examinees each with abilities $-2.229,-0.171$ for Item 2 and 1.035, 2.965 for Item 3. The restricted optimal design suggests us to select $18.78 \%$, $10.14 \%$ and $14.29 \%$ of the population of examinees on the ability intervals $(-4.069,-0.886)$, $(-0.329,-0.069)$ and $(0.338,0.757)$, respectively, for Item 2 . It also recommends to choose $16.01 \%, 5.05 \%$ and $15.72 \%$ of examinees from the population on the ability intervals $(-0.069$, $0.338),(0.757,0.938)$ and $(1.006,5.508)$ for Item 3 , see Fig. 9. The directional derivative plot in the lower panel of Fig. 9 shows together with the Equivalence Theorem for Item Calibration that this design is optimal for the selection of examinees: We select examinees on intervals below the blue line for Item 2 or 3 depending on which item's directional derivative is smallest in these intervals. In contrast to the preceding example, the competition between the items leads here to the need of three intervals for each item. Note that in the region from $\theta=-0.3$ to 0.9 , the two directional derivatives are quite close but do not exactly coincide - the minimum is unique except for the crossing points. Optimal designs for other values of $s \in\{0.1,0.2, \ldots, 1\}$ are presented in Fig. 10. 
TABLE 3

Relative efficiency $\mathrm{RE}_{\mathrm{D}}$ versus D-optimal restricted design for calibration of two or more items.

\begin{tabular}{|c|c|c|c|c|c|c|}
\hline \multirow[t]{2}{*}{ Proportion $(\%)$} & \multicolumn{2}{|c|}{ Item 1,2} & \multicolumn{2}{|c|}{ Item 1,3} & \multicolumn{2}{|c|}{ Item 2,3} \\
\hline & $\mathrm{RE}_{\mathrm{D}}$ & $\mathrm{RE}_{\mathrm{SS}}(\%)$ & $\mathrm{RE}_{\mathrm{D}}$ & $\mathrm{RE}_{\mathrm{SS}}(\%)$ & $\mathrm{RE}_{\mathrm{D}}$ & $\operatorname{RE}_{\mathrm{SS}}(\%)$ \\
\hline \multicolumn{7}{|c|}{ (a) Relative efficiency of random design versus D-optimal restricted design } \\
\hline 0 & 0.6884 & 45.2694 & 0.5191 & 92.6486 & 0.4796 & 108.5273 \\
\hline 10 & 0.6915 & 44.6164 & 0.5528 & 80.8993 & 0.5113 & 95.5664 \\
\hline 20 & 0.6997 & 42.9275 & 0.5896 & 69.6201 & 0.5444 & 83.6903 \\
\hline 30 & 0.7107 & 40.7010 & 0.6226 & 60.6242 & 0.5748 & 73.9856 \\
\hline 40 & 0.7238 & 38.1549 & 0.6531 & 53.1150 & 0.6032 & 65.7839 \\
\hline 50 & 0.7404 & 35.0594 & 0.6821 & 46.5996 & 0.6304 & 58.6238 \\
\hline 60 & 0.7597 & 31.6309 & 0.7104 & 40.7728 & 0.6585 & 51.8552 \\
\hline 70 & 0.7810 & 28.0483 & 0.7399 & 35.1514 & 0.6871 & 45.5363 \\
\hline 80 & 0.8037 & 24.4270 & 0.7715 & 29.6245 & 0.7137 & 40.1228 \\
\hline 90 & 0.8275 & 20.8469 & 0.8048 & 24.2492 & 0.7392 & 35.2876 \\
\hline 100 & 0.8525 & 17.2983 & 0.8398 & 19.0805 & 0.7665 & 30.4547 \\
\hline \multirow[t]{2}{*}{ Proportion (\%) } & \multicolumn{2}{|c|}{ Item 1,2} & \multicolumn{2}{|c|}{ Item 2, 3} & & \\
\hline & $R E_{D}$ & $\mathrm{RE}_{\mathrm{SS}}(\%)$ & $R E_{D}$ & $\mathrm{RE}_{\mathrm{SS}}(\%)$ & & \\
\hline \multicolumn{7}{|c|}{ (b) Relative efficiency of symmetric design versus D-optimal restricted design } \\
\hline 0 & 1.0000 & 0.0000 & 1.0000 & 0.0000 & & \\
\hline 10 & 0.9996 & 0.0370 & 0.9796 & 2.0784 & & \\
\hline 20 & 0.9972 & 0.2772 & 0.9571 & 4.4781 & & \\
\hline 30 & 0.9930 & 0.7070 & 0.9404 & 6.3430 & & \\
\hline 40 & 0.9885 & 1.1670 & 0.9289 & 7.6589 & & \\
\hline
\end{tabular}

$\mathrm{RE}_{\mathrm{SS}}$ indicates sample size gain when using optimal restricted design instead of symmetric design.

\subsection{Calibration of Two Items Using the Whole Population}

Now we want to select all the available examinees in order to calibrate Item 2 and 3 in the item bank. The optimal unrestricted design suggests to choose $25 \%$ each of all available examinees at the ability levels $-2.229 \&-0.171$ for Item 2 and $25 \%$ each for Item 3 at the ability levels 1.035 $\& 2.965$. When we use restricted optimal design, we should choose $30.98 \%$ of the examinees on the ability interval $(-\infty,-0.496)$ and $23.28 \%$ on $(0.081,0.723)$ for Item 2 . For Item 3 , it suggests to choose $22.27 \%$ examinees on $(-0.496,0.081)$ and $23.47 \%$ on $(0.723, \infty)$. (With an exact computation, the last interval is obtained to be $(0.723,10)$; examinees with higher ability should receive Item 2 . However, the probability for an ability $\geq 10$ is basically 0 .) The directional derivative in the third panel of Fig. 9 shows that this design is optimal for selection of examinees: We choose examinees for Item 2 or 3 whenever the directional derivative is smallest. The random design requires $30.45 \%$ more examinees to be as efficient as the locally D-optimal restricted design.

\subsection{Calibration of All Three Items Using the Whole Population}

Finally, we calibrate Item 1, 2 and 3 simultaneously using the population of examinees participating in the computerized test. The optimal unrestricted design recommends us to select approximately $16.67 \%$ of all available examinees at each of six optimal unrestricted design points of ability. For the optimal restricted design, we remarked in Sect. 2.3 that examinees with very high abilities should be assigned to the item with the lowest discrimination, here Item 1 . For numerical computation, we assign therefore intervals $I_{10}=\left(-\infty, \theta_{10 U}\right]$ and $I_{1(K+1)}=\left[\theta_{1(K+1) L}, \infty\right)$ 
to Item 1; between these intervals, we calculate an optimal $K$-interval design. It turns out that $K=2$ is sufficient here. The optimal restricted design suggests us to choose $11.97 \%$ and $23.17 \%$ of the total available examinees on the ability interval $(-5.147,-1.176)$ and $(-0.424,0.170)$ for Item 2 . Besides the intervals $(-\infty,-5.147)$ and $(5.975, \infty)$ where almost no examine falls in, we choose $21.62 \%$ and $16.02 \%$ of examinees for Item 1 on the intervals $(-1.176,-0.424)$ and $(0.754,1.513)$. Lastly, on the intervals $(0.170,0.754)$ and $(1.513,5.975)$ we select $20.70 \%$ and $6.82 \%$ of examinees for Item 3, see forth panel of Fig. 11. According to the Equivalence Theorem for Item Calibration the directional derivatives in the last panel of Fig. 11 show that the restricted design is optimal for selection of examinees based on their estimated abilities. The random design needs $32.04 \%$ more examinees to have the same efficiency as the restricted D-optimal design.

An alternative way of calibration would be the administration of all three items to all available examinees. The main "cost" of it is the increased testing time for each examinee which is three times larger (if we make the simplifying assumption that all items require the same testing time). The information from this design is three times the information of the random design and we can therefore use efficiencies with respect to the random design to compute efficiency of the allexaminees-all-items design versus the restricted D-optimal design. As written above, the restricted D-optimal design has $32.04 \%$ sample gain, or in other words, it has 1.3204 times the information of the random design. Therefore, the all-examinees-all-items design has $3 / 1.3204=2.27$ times the information of the restricted D-optimal design despite needing three times more time. Since one has in reality more than three items to calibrate (see Sect. 5), the time gain is usually important to achieve.

\section{Scaling Up the Method for Large Banks of New Items}

An assumption we made was that each examinee can calibrate (at most) one item. We had examples with an item bank of three items. Realistic situations have often large banks of new items, and it is desired that each examinee calibrates several items. We will show now how one can easily use the methods described for realistic situations. We assume that the maximal number $k$ of items which an examinee can calibrate is given by practical circumstances, e.g., the time necessary for the test. The number of new items $n$ to calibrate is $>k$, such that we need to allocate them to different examinees. Let us assume for simplicity that $n$ is a multiple of $k$. We divide the $n$ items into $k$ blocks of $n / k$ items each. Each examinee is supposed to calibrate exactly one item per block. The blocking might be done taking content of items into account or simply randomly. We compute now for each of the $n / k$-item block the optimal restricted design separately. This gives us the optimal calibration with the additional restriction of this blocking.

We can compute the D-efficiency of the random design compared to the restricted optimal design for each block. It follows from formula (10) that the overall efficiency of the random design compared to the blocked restricted optimal design is the geometric mean of the block efficiencies.

\section{Discussion and Conclusion}

Item calibration is an important tool for maintaining, updating and developing new items for an item bank. In the case of a two-parameter logistic model, the unrestricted D-optimal design for calibration of one new item has two optimal ability levels of examinees $\left(\theta=b \pm \frac{1.543}{a}\right)$ where one should sample equal proportions of the examinee population at these points. In practice, it is impossible to sample equal proportions of examinees from these optimal points of ability due to unavailability or limited availability of examinees. Sampling symmetrically around the optimal ability levels works in some situations. But in many cases, it is not clear how to define such 

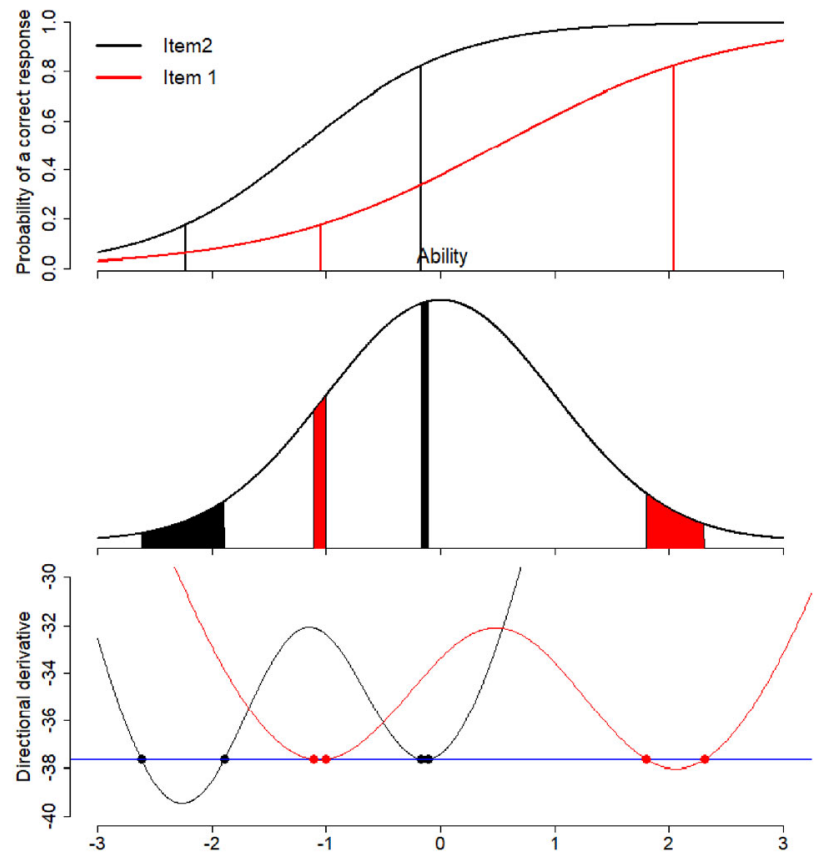

(a) Calibration of Item 1 and 2 by $10 \%$ of the examinees. Upper panel: black and red lines represent the two-parameter logistic model curves. The vertical lines indicate the ability levels of the locally D-optimal unrestricted design; Middle panel: two shaded red and black parts of normal distribution represent the intervals for ability levels of the locally D-optimal restricted design for these items; Lower panel: Black and red line curves represent the directional derivatives while black and red dots marked on it depict the lower and upper interval limits of the locally D-optimal restricted design of these items. The blue line is a reference line and where we sample when the directional derivative is below in this line.

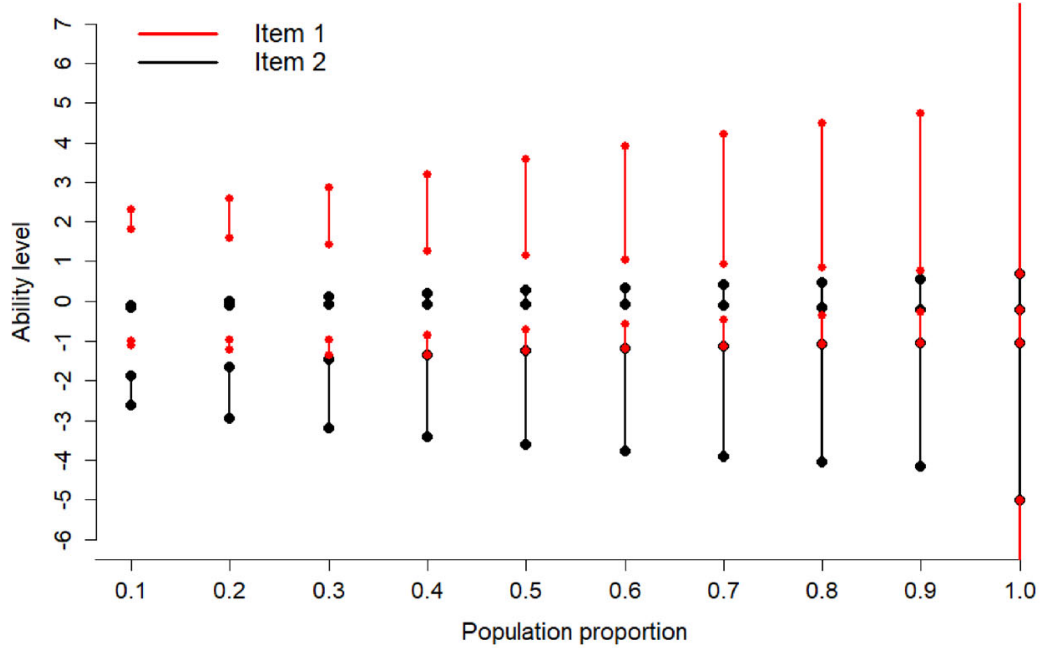

(b) Locally D-optimal restricted designs for sample proportion $s=0.1,0.2, \ldots, 1$

FIGURE 7.

Locally D-optimal restricted designs for simultaneous calibration of Item 1 and 2. 


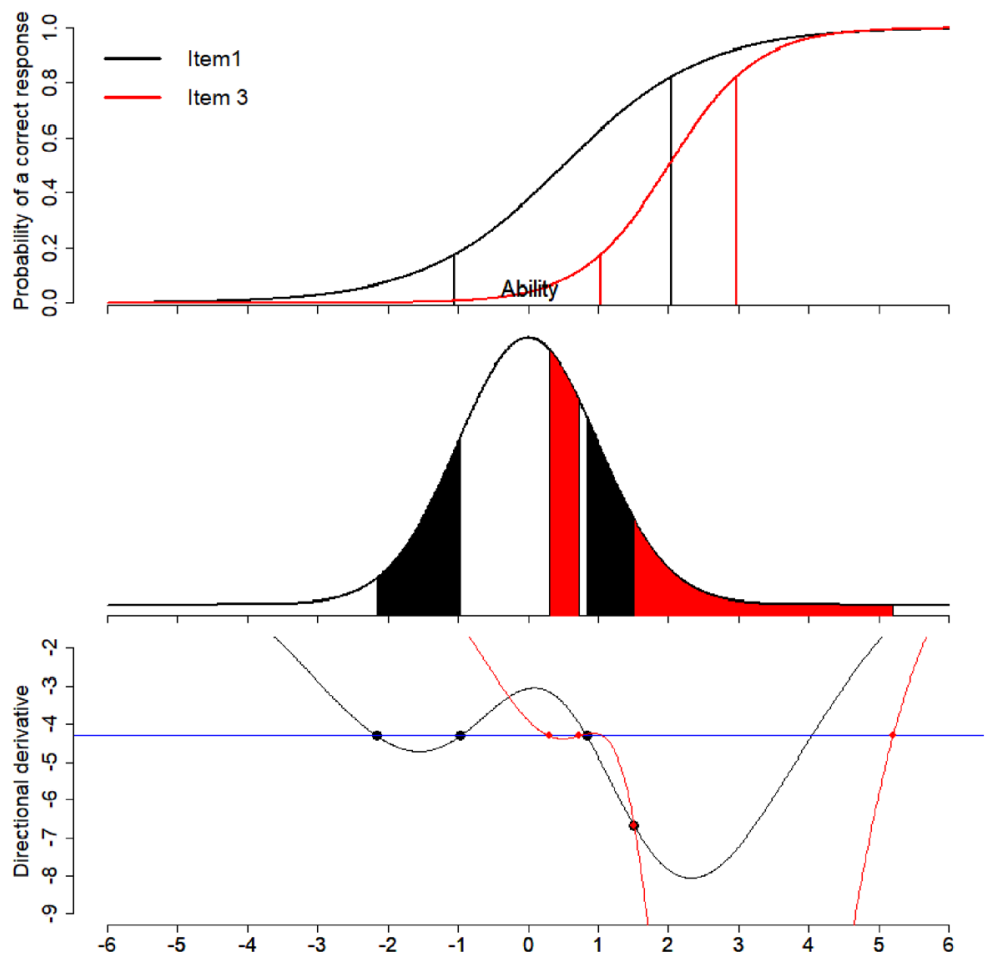

(a) Calibration of Item 1 and 3 by $50 \%$ of examinees; description see Figure 7 a.

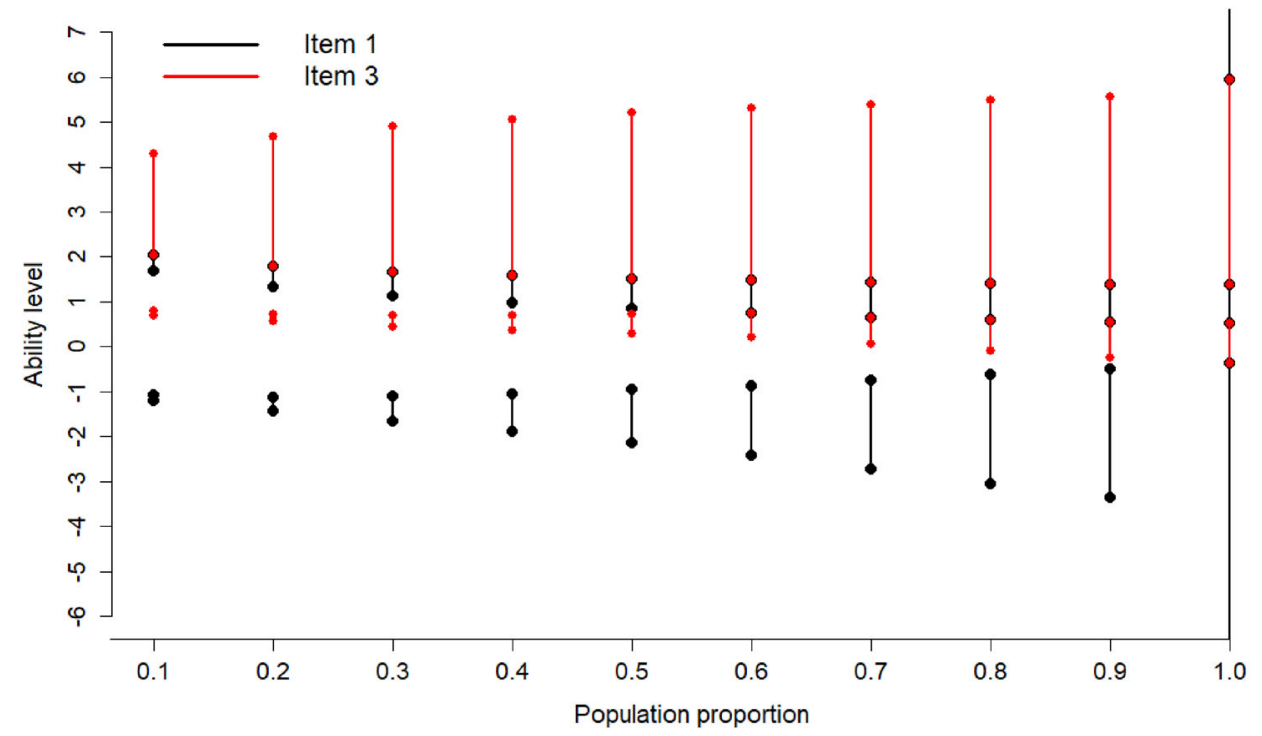

(b) Locally D-optimal restricted designs for sample proportion $s=0.1,0.2, \ldots, 1$.

FIGURE 8.

Locally D-optimal restricted designs for simultaneous calibration of Item 1 and 3. 

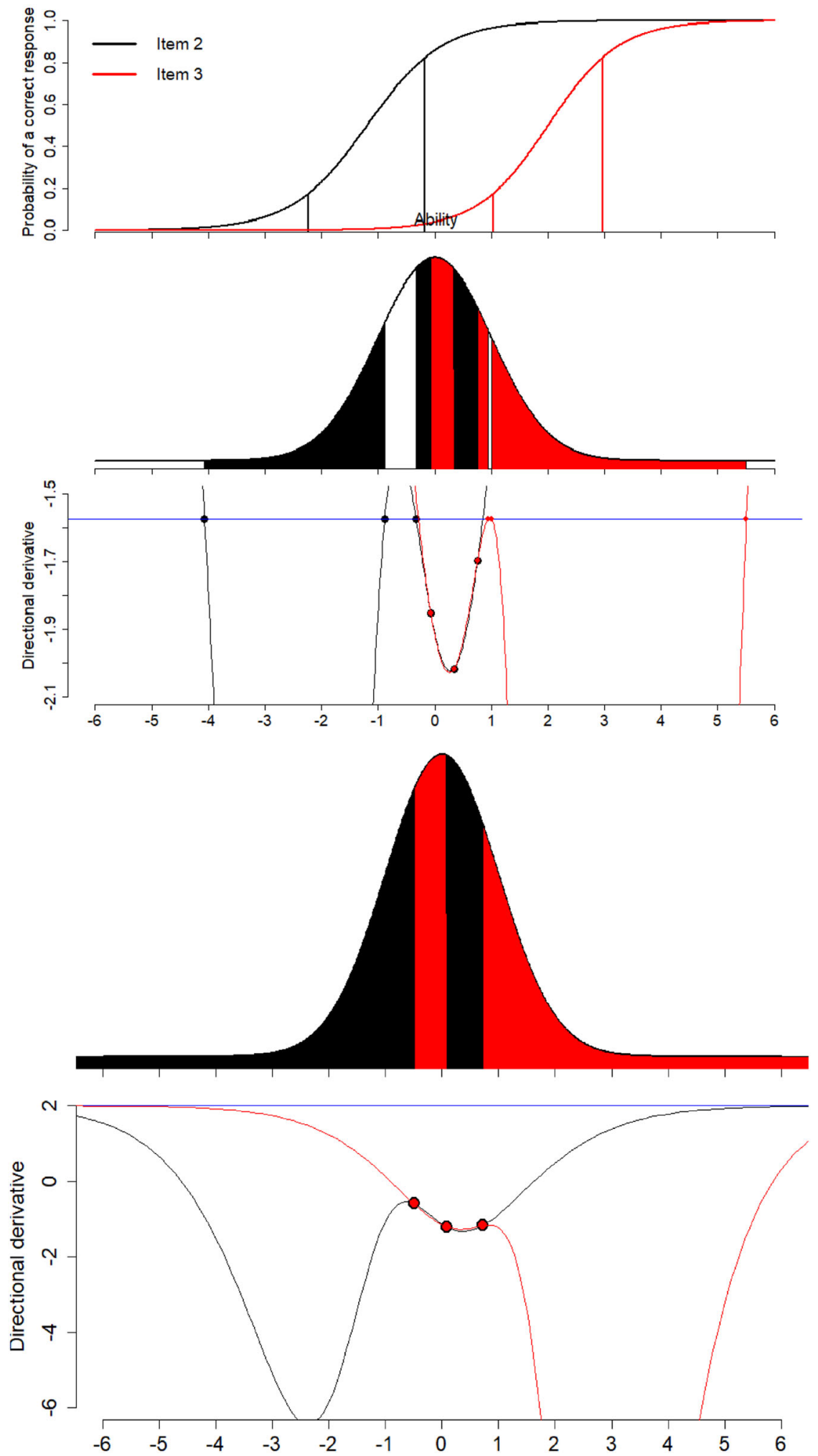

FIGURE 9.

Calibration of Item 2 and 3 using $80 \%$ of examinees and the whole population of examinees, see Fig. 7 a. 


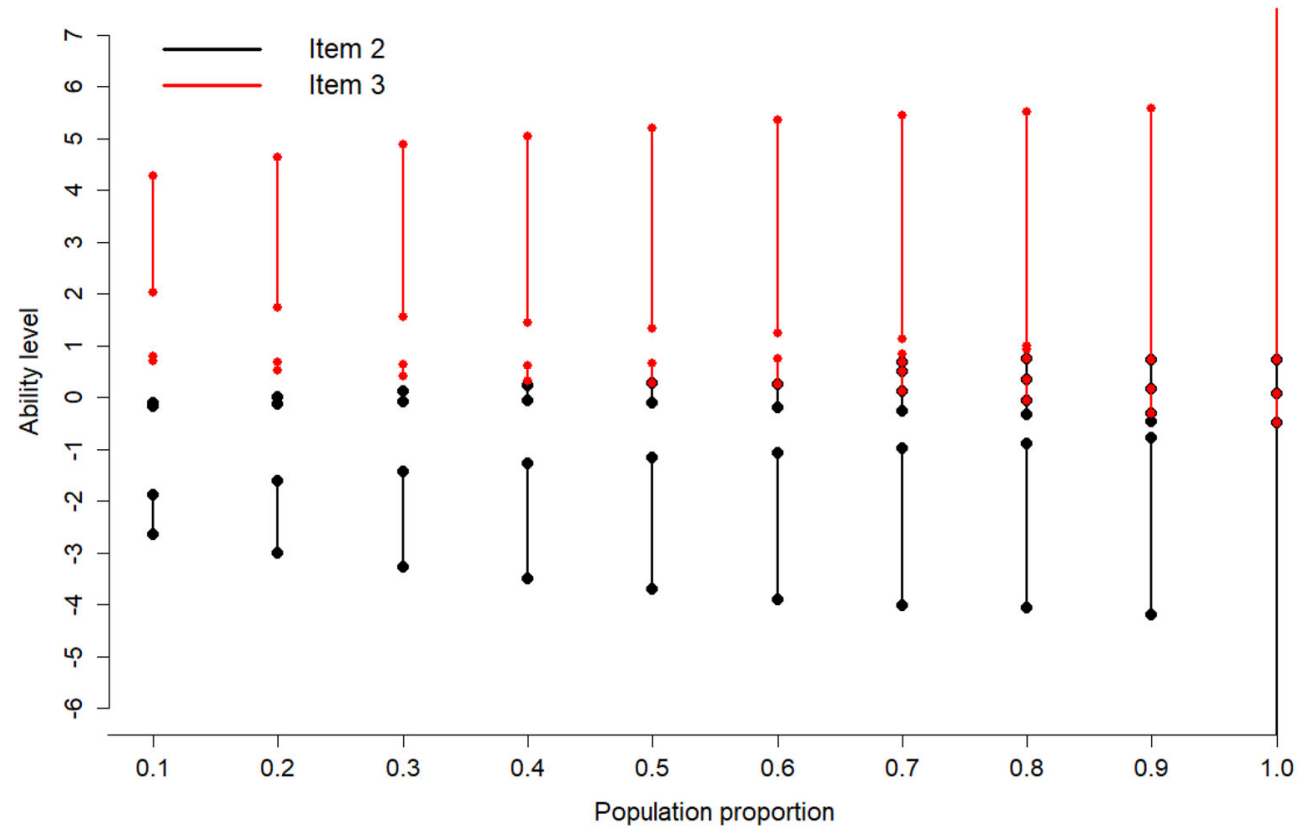

FIGURE 10.

Locally D-optimal restricted designs for simultaneous calibration of Item 2 and 3 for sample proportion $s=0.1,0.2, \ldots, 1$.

symmetric designs, e.g., if optimal ability levels are too close to each other. To avoid possibly inefficient ad hoc solutions, we have used restricted optimal designs to calibrate new items where we used optimal intervals instead of points to sample the examinees from the population.

In this paper, we derived locally optimal designs. Their quality might depend on the quality of the prior guess about the item parameters. If the true item parameters are a little different from the prior guess, we have seen robustness; however, if the difference is large, the locally optimal design might be a bad choice. Therefore, alternatives to local optimality, Bayesian or maximin optimality, can be applied, see e.g., Atkinson et al. (2007), Chapter 17 and 18. Combination of these general optimal design approaches with the restricted optimality considered here could be an area of future research.

Further, an opportunity in computerized calibration is to re-estimate the item parameters from the ongoing calibration and to apply a sequential optimal design, see Lu (2014), van der Linden and Ren (2015) and Ren et al. (2017). This sequential and the Bayesian (or minimax) approach can also be combined. However, in tests, e.g., the Swedish Scholastic Assessment Test, all examinees are tested more or less simultaneously. If calibration items are added to tests where all examinees are tested more or less in parallel, we think therefore that a minimax or Bayesian approach should be used in a non-sequential context.

In this manuscript, we assume that abilities of examinees are well determined in the operational part of the test before it is decided which item to calibrate depending on their ability. We ignore here the fact that we use estimated abilities and not true abilities, but there is some uncertainty around the estimates: The examinee might be a bit better or worse than the estimated ability (the examinee might have had bad or good luck in the examination). However, the abilities should be reasonably well estimated if the operational part of the achievement test is large and calibration items are added toward the end of this test. Note that Ren et al. (2017) suggested to seed the new items into the final part of the test and $\mathrm{He}$ et al. (2019) concluded in their situation 


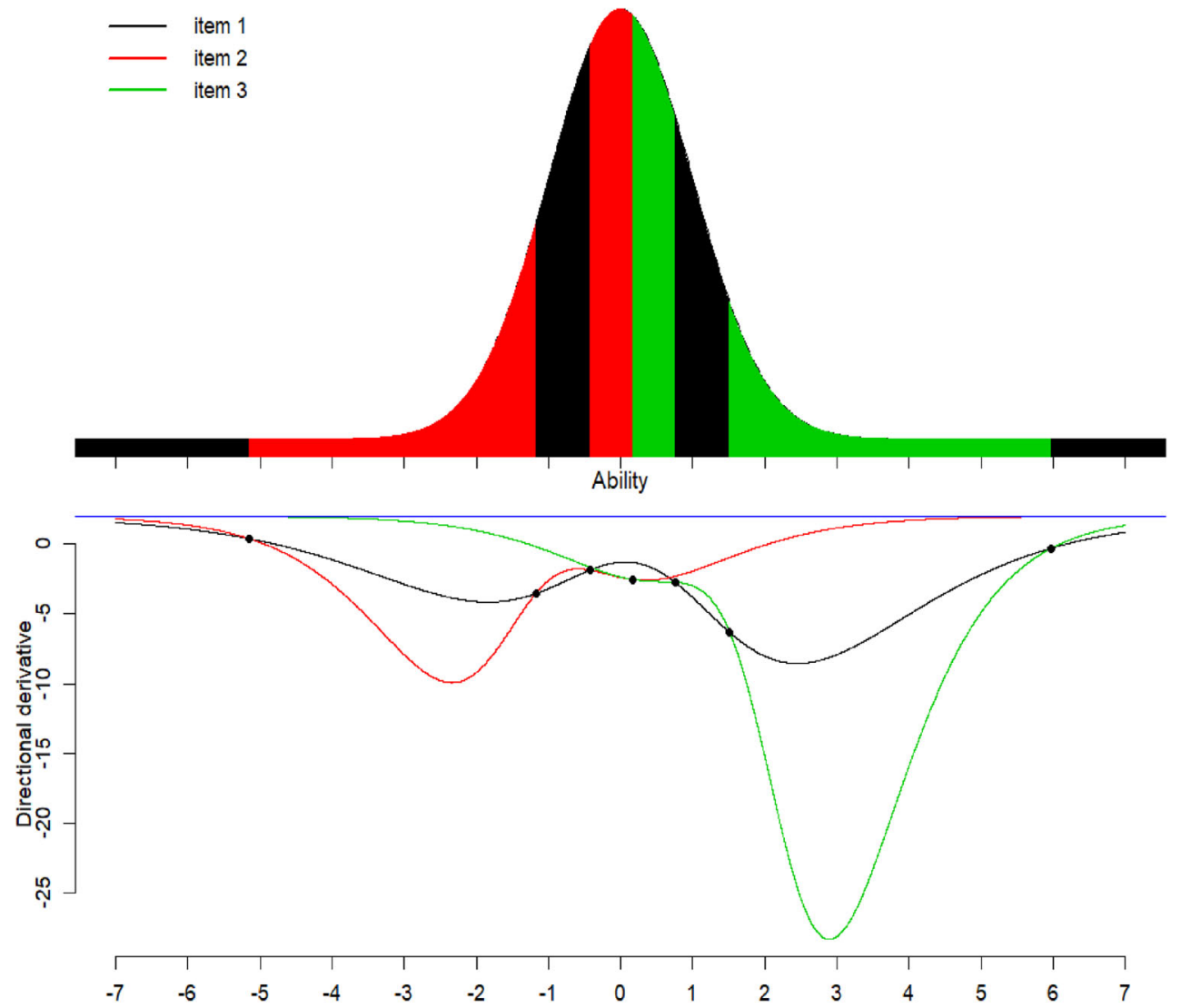

FIGURE 11.

Calibration of Item 1, 2 and 3 by all examinees, see Fig. 7a.

that even middle positions worked equally well. Nevertheless, for handling the uncertainty in abilities, it is conceptually possible to use the here described restricted optimal design approach in connection with using posterior distributions of abilities [see e.g., Section 2.1. of Ren et al. (2017)] rather than point estimates.

While our developed theory applies generally to item response models and to convex and differentiable optimality criteria, we have in the examples considered a two-parametric logistic model together with D-optimality. It might be interesting to explore the structure of optimal designs for other models. For example including a third parameter modeling a guessing probability has been advocated in this context of achievement tests, see e.g., van der Linden and Ren (2015). Further, the examinees' abilities might not adequately be characterized by a one-dimensional ability parameter. Then a multidimensional IRT model might be considered. Optimal estimated designs for these models will be considered in future research where other optimality criteria will be considered as well.

Finally, we assumed in the Equivalence Theorem for Item Calibration that each examinee at most can calibrate one item. We described how this can be applied in a situation where everyone calibrates more items. This leads however to an optimal design under a blocking restriction. When there is no content-reason for a specific blocking and when the blocks are created randomly, it might be desirable to improve the design even more and to drop the blocking restriction. An 
extension of the Equivalence Theorem such that optimization can be done without the blocking restriction is a task for future research.

\section{Acknowledgments}

We would like to thank Professor Daniel Thorburn from our department for discussions about this topic and two unknown referees for their comments which improved our manuscript.

Open Access This article is distributed under the terms of the Creative Commons Attribution 4.0 International License (http://creativecommons.org/licenses/by/4.0/), which permits unrestricted use, distribution, and reproduction in any medium, provided you give appropriate credit to the original author(s) and the source, provide a link to the Creative Commons license, and indicate if changes were made.

Publisher's Note Springer Nature remains neutral with regard to jurisdictional claims in published maps and institutional affiliations.

\section{A. Appendix: Proof of Theorem in Sect. 2.3}

We have to prove: " $h^{*}$ is $\Psi$-optimal in $\Xi_{s}^{g} \Leftrightarrow(7)$ ". Condition (7) means: $P_{h^{*}}(\{(\theta, i) \in \tilde{\chi}$ : $\left.\left.F_{\Psi}\left(h^{*}, \theta, i\right)>L\left(h^{*}, \theta\right)\right\}\right)=0$.

“Æ” Let $\tilde{h} \in \Xi_{s}^{g}$ be arbitrary. We have

$$
F_{\Psi}\left(h^{*}, \tilde{h}\right)=\sum_{i=1}^{n} F_{\Psi}\left(h^{*}, \tilde{h}_{i}\right)=\sum_{i=1}^{n} \int_{\Theta} F_{\Psi}\left(h^{*}, \theta, i\right) \tilde{h}_{i}(\theta) \mathrm{d} \theta .
$$

Since $F_{\Psi}(h, \theta, i) \geq L(h, \theta)$, we have

$$
F_{\Psi}\left(h^{*}, \tilde{h}\right) \geq \sum_{i=1}^{n} \int_{\Theta} L\left(h^{*}, \theta\right) \tilde{h}_{i}(\theta) \mathrm{d} \theta=\int_{\Theta} L\left(h^{*}, \theta\right) \sum_{i=0}^{n} \tilde{h}_{i}(\theta) \mathrm{d} \theta-\int_{\Theta} L\left(h^{*}, \theta\right) \tilde{h}_{0}(\theta) \mathrm{d} \theta
$$

Since $L \leq c^{*} h^{*}$-almost surely and the integral over $\tilde{h}_{0}$ is $1-s$,

$$
\begin{aligned}
F_{\Psi}\left(h^{*}, \tilde{h}\right) & \geq \int_{\Theta} L\left(h^{*}, \theta\right) g(\theta) \mathrm{d} \theta-c^{*}(1-s)=\int_{\Theta} L\left(h^{*}, \theta\right) \sum_{i=1}^{n} h_{i}^{*}(\theta) \mathrm{d} \theta \\
& \stackrel{(7)}{=} \sum_{i=1}^{n} \int_{\Theta} F_{\Psi}\left(h^{*}, \theta, i\right) h_{i}^{*}(\theta) \mathrm{d} \theta=\sum_{i=1}^{n} F_{\Psi}\left(h^{*}, h_{i}^{*}\right)=F_{\Psi}\left(h^{*}, h^{*}\right)=0
\end{aligned}
$$

(the last equation follows directly from the definition of the directional derivative). This means that we have shown $F_{\Psi}\left(h^{*}, \tilde{h}\right) \geq 0$ for any design $\tilde{h} \in \Xi_{s}^{g}$ and the optimality of $h^{*}$ follows from a theorem of Whittle (1973), see also Theorem 3.6 of Silvey (1980) and Theorem A (i) of Sahm and Schwabe (2000).

" $\Rightarrow$ " We will show this implication by contradiction. If (7) is not fulfilled, then there is some $j \in\{0,1, \ldots, n\}$ with $P_{h_{j}^{*}}\left(\left\{\theta: F_{\Psi}\left(h^{*}, \theta, j\right)>L\left(h^{*}, \theta\right)\right\}\right)>0$ meaning that there are some $j, k \in\{0,1, \ldots, n\}, j \neq k$, with $P_{h_{j}^{*}}\left(\left\{\theta: F_{\Psi}\left(h^{*}, \theta, j\right)>F_{\Psi}\left(h^{*}, \theta, k\right)\right\}\right)>0$. Case $1: j, k \in$ $\{1, \ldots, n\}$. There has to be some set $\Theta_{0} \subseteq \Theta$ with $F\left(h^{*}, \theta, j\right)>F\left(h^{*}, \theta, k\right)$ for all $\theta \in \Theta_{0}$ and 
$P_{h_{j}^{*}}\left(\Theta_{0}\right)>0$, i.e., item $j$ is sampled on $\Theta_{0}$ despite there is another item, $k$, which has a smaller directional derivative. We construct a new design $h^{* *}$ by moving all sample mass on $\Theta_{0}$ from item $j$ to item $k$ :

$$
\begin{aligned}
h^{* *}(\theta, i) & =h^{*}(\theta, i) \text { if } \theta \in \Theta \backslash \Theta_{0} \text { or } i \notin\{j, k\}, \\
h^{* *}(\theta, k) & =h^{*}(\theta, k)+h^{*}(\theta, j) \text { and } h^{* *}(\theta, j)=0 \text { if } \theta \in \Theta_{0} .
\end{aligned}
$$

It is clear that $h^{* *} \in \Xi_{s}^{g}$. We have then

$$
F_{\Psi}\left(h^{*}, h^{* *}\right)=F_{\Psi}\left(h^{*}, h^{*}\right)+\int_{\Theta_{0}}\left\{F_{\Psi}\left(h^{*}, \theta, k\right)-F_{\Psi}\left(h^{*}, \theta, j\right)\right\} h^{* *}(\theta, j) \mathrm{d} \theta<0
$$

where the inequality holds since $F\left(h^{*}, h^{*}\right)=0$ and since the integral is negative due to the fact that $F_{\Psi}\left(h^{*}, \theta, k\right)-F_{\Psi}\left(h^{*}, \theta, j\right)$ is negative. Since we have found an $h^{* *} \in \Xi_{S}^{g}$ with $F_{\Psi}\left(h^{*}, h^{* *}\right)<0$, it follows that the design $h^{*}$ cannot be $\Psi$-optimal in $\Xi_{s}^{g}$, according to the theorem of Whittle (1973). Case 2: $j=0, k \in\{1, \ldots, n\}$. There has to be some set $\Theta_{0} \subseteq \Theta$ with $c^{*}>F_{\Psi}\left(h^{*}, \theta, k\right)$ for all $\theta \in \Theta_{0}$ and $P_{h_{0}^{*}}\left(\Theta_{0}\right)>0$, i.e., there is some positive non-sample probability where the directional derivative of item $k$ is below $c^{*}$. From the definition of $c^{*}$ follows that if one samples everywhere where $\tilde{L}<c^{*}$, then the sampling mass is $\leq s$. Therefore, if no full sampling is done on $\Theta_{0} \subseteq\left\{\theta: L<c^{*}\right\}$, there has to be sampling on $\left\{\theta: L=c^{*}\right\}$. Formally, this means that we can find $\Theta_{1} \subseteq\left\{\theta: L=c^{*}\right\}$ such that $\int_{\Theta_{0}} h_{0}(\theta) \mathrm{d} \theta=\int_{\Theta_{1}}\left(g(\theta)-h_{0}(\theta)\right) \mathrm{d} \theta$. We can then construct a competing design $\xi^{* *}$ by moving sampling mass from $\Theta_{1}$ to item $k$ on $\Theta_{0}$ :

$$
\begin{aligned}
h^{* *}(\theta, 0) & =\sum_{i=0}^{n} h^{*}(\theta, i) \text { and } h^{* *}(\theta, 1)=\cdots=h^{* *}(\theta, n)=0 \text { if } \theta \in \Theta_{1}, \\
h^{* *}(\theta, k) & =h^{*}(\theta, k)+h^{*}(\theta, 0) \text { and } h^{* *}(\theta, 0)=0 \text { if } \theta \in \Theta_{0}, \\
h^{* *} & =h^{*}, \text { otherwise. }
\end{aligned}
$$

One can show $h^{* *} \in \Xi_{s}^{g} ; F_{\Psi}\left(h^{*}, h^{* *}\right)<0$. Non-optimality of $h^{*}$ follows like in Case 1 .

Case 3: $j \in\{1, \ldots, n\}, k=0$. A competing design $h^{* *}$ with $F_{\Psi}\left(h^{*}, h^{* *}\right)<0$ can be constructed similarly like in Case 2.

\section{References}

Abdelbasit, K. M., \& Plackett, R. L. (1983). Experimental design for binary data. Journal of the American Statistical Association, 78(381), 90-98.

Atkinson, A. C., Donev, A. N., \& Tobias, R. D. (2007). Optimum experimental designs, with SAS. Oxford: Oxford University Press.

Berger, M. P. F. (1992). Sequential sampling designs for the two-parameter item response theory model. Psychometrika, 57(4), 521-538.

Berger, M. P. F., King, C. J., \& Wong, W. K. (2000). Minimax D-optimal designs for item response theory models. Psychometrika, 65(3), 377-390.

Berger, M. P. F., \& Wong, W. K. (2009). An introduction to optimal designs for social and biomedical research (Vol. 83). Chichester: Wiley.

Borchers, H. W. (2013). nloptwrap: Wrapper for package nloptr. R package version 0.5-1. http://CRAN.R-project.org/ package=nloptwrap. Accessed 30 July 2018.

Buyske, S. (1998). Optimal design for item calibration in computerized adaptive testing: The 2PL case. In N. Flournoy, W. F. Rosenberger, \& W. K. Wong (Eds.), New developments and applications in experimental design. Berkeley: Institute of Mathematical Statistics.

Buyske, S. (2005). Optimal designs in educational testing. In M. P. F. Berger \& W. K. Wong (Eds.), Applied optimal designs. Chichester: Wiley. 
Chang, Y.-C. I., \& Lu, H.-Y. (2010). Online calibration via variable length computerized adaptive testing. Psychometrika, $75(1), 140-157$.

He, Y., Chen, P., \& Li, Y. (2019). New efficient and practicable adaptive designs for calibrating items online. Applied Psychological Measurement. https://doi.org/10.1177/0146621618824854.

Jones, D. H., \& Jin, Z. (1994). Optimal sequential designs for on-line item estimation. Psychometrika, 59(1), 59-75.

Kiefer, J., \& Wolfowitz, J. (1960). The equivalence of two extremum problems. Canadian Journal of Mathematics, 12(5), 363-365.

Lu, H.-Y. (2014). Application of optimal designs to item calibration. PLoS ONE, 9(9), e106747.

Pukelsheim, F. (2006). Optimal design of experiments. Philadelphia: SIAM.

Ren, H., van der Linden, W. J., \& Diao, Q. (2017). Continuous online item calibration: Parameter recovery and item utilization. Psychometrika, 82(2), 498-522.

Sahm, M., \& Schwabe, R. (2000). A note on optimal bounded designs. In A. Atkinson, B. Bogacka, \& A. A. Zhigljavsky (Eds.), Optimum Design 2000 (pp. 131-140). Dordrecht: Kluwer Academic Publishers.

Silvey, S. D. (1980). Optimal design. Monographs on applied probability and statistics (1st ed., Vol. 1). London: Chapman and Hall.

Stocking, M. L. (1988). Scale drift in on-line calibration. New York: Wiley Online Library.

Universitets- och högskolerådet (2019). Studera.nu. Provdagen - så fungerar det. http://www.studera.nu/hogskoleprov/ infor-hogskoleprovet/provdagen-sa-fungerar-det/. Accessed 30 July 2018.

van der Linden, W. J., \& Ren, H. (2015). Optimal Bayesian adaptive design for test-item calibration. Psychometrika, 80(2), 263-288.

Whittle, P. (1973). Some general points in the theory of optimal experimental design. Journal of the Royal Statistical Society. Series B (Methodological), 35(1), 123-130.

Wynn, H. P. (1982). Optimum submeasures with applications to finite population sampling. Statistical decision theory and related topics III (pp. 485-495). New York: Academic Press.

Zheng, Y. (2014). New methods of online calibration for item bank replenishment. PhD thesis, University of Illinois at Urbana-Champaign.

$\mathrm{Zhu}, \mathrm{R}$. (2006). Implementation of optimal design for item calibration in computerized adaptive testing (CAT). $\mathrm{PhD}$ thesis, Champaign, IL, USA.

Manuscript Received: 31 JUL 2018

Final Version Received: 28 MAY 2019

Published Online Date: 10 JUN 2019 